\title{
Link Quality Prediction in Mobile Ad-Hoc Networks
}

\author{
Gregor Gaertner and Eamnn O'Nuallain \\ School of Computer Science and Statistics, \\ Trinity College Dublin, \\ Ireland
}

\section{Introduction}

Cognitive radio is the radio revolution of our time. With it comes the promise of huge swathes of unused bandwidth which will provide the fuel for sophisticated wireless applications and fast and reliable wireless internet use. It is as yet unclear precisely what form cognitive networks will take. They may be infrastructure-based, ad-hoc or some combination of the two resulting from a trade-off between technological ideals and economic imperatives. With regard to ad-hoc networks it is clear that they will, at the very least, form an important part of cognitive radio networks for without them co-operative sensing would be considerably less effective. Cooperative sensing is an important tool in guaranteeing non-interference. Indeed in areas or at times where there is no radio infrastructure ad-hoc networks are essential. Communication between ad-hoc nodes is a vexed problem. The propagation environment may be harsh and/or changeable. Furthermore it is very difficult to predict accurately what the signal strength at an intended receiver is. Of course propagation models abound but none can accurately predict signal strength without recourse to topological data and even then these are slow to compute. This is why researchers have focused on path-loss models or statistical methods with which to make their predictions. The following is a literature review of the most prominent methods which have been published over the last fifteen years which will convey for the reader the coalface of research in this area.

Wireless mobile ad hoc networks (MANETs) (Schiller 2000, pp. 275-286) are self-organizing communication networks without any infrastructure. Peer nodes work collaboratively to transport packets through the network in a store and forward fashion since the limited transmission radius of nodes necessitates multi-hop communication. MANETs are appropriate in scenarios in which an infrastructure is either not feasible due to economic constraints or not available due to physical constraints such as natural disasters or battlefield deployments. Applications range from the communication-enabled soldier, disaster recovery and Voice over IP to mobile gaming.

The mobility of nodes and radio propagation effects cause frequent changes in the topology of a MANET; link failures and link recoveries, which are infrequent events in wired networks, occur frequently in MANETs. Consequently, reliable high-bandwidth communication is a challenge that can only partially be addressed by existing methods for wired networks. One successful strategy to alleviate the impact of topology changes is to 
predict them such that corrective actions can be taken before the change occurs (e.g. Goff et al. 2001). In the literature the effectiveness of such proactive operations has been demonstrated in the area of multicast communications and routing, in which packet latency is significantly reduced by discovering new routes before links fail. Similar performance enhancements are envisioned for group communications with partition prediction. Link quality prediction (LQP) is the foundation for proactive operations and is therefore a key technology for the efficient operation of MANETs.

\section{Link quality prediction algorithms}

LQP algorithms can be classified using a variety of criteria. One criterion is the type of input that the algorithm uses to make predictions. The inputs can be signal-power measurements, location measurements, or other measurements such as the ratio of transmitted to received packets within some time interval. In fact, the type of input is the most significant criterion by which to classify LQP algorithms since it typically represents the focus of the algorithms' creators. Predictions based on signal-power measurements focus on the radio propagation model while predictions based on location measurements concentrate on the mobility model. Another criterion by which to classify existing algorithms is the output of the LQP. If the output is subset of a finite set of states, we classify the prediction as being deterministic. Most deterministic prediction algorithms surveyed have only two possible output states; packets are predicted to be either lost or received for some time into the future. If the output of an LQP algorithm is a subset of an infinite set of states, we classify this prediction as stochastic since the output is in that case usually a probability. For example, an algorithm that estimates the probability of successfully receiving future transmitted packets is stochastic. Another possible classification criterion is the application of LQP. Successful applications are found in the area of routing, group communication, multicast communication and clustering. A final possible classification criterion is the radio propagation model on which a LQP algorithm is based. Since link quality is primarily dependent on the radio propagation model, even algorithms that focus on location measurements and mobility models are explicitly or implicitly derived with respect to a specific radio propagation model. In the literature we commonly found the use of the simple Radial Propagation Model, the Free Space Propagation Model (Rappaport 2002, pp. 107109) and the Two-Ray Ground Propagation Model (Rappaport 2002, pp. 120-125). In the simple Radial Propagation Model a node ' $i$ ' can communicate with a node ' $j$ ' if the distance between ' $i$ ' and ' $j$ ' is less than or equal to a threshold; otherwise ' $i$ ' does not receive packets that are sent by ' $j$ ' and vice versa.

A graphical overview of the taxonomy of the surveyed literature based on these criteria is presented in Figure 1. Note that Roman et al.'s (2001) and Killijian et al.'s (2001) works are of a conceptual nature only such that we could only assess them with respect to their applications. Apart from these two studies all other works have been classified according to their input parameters, output parameters, radio propagation model and application area. Punnoose et al.'s (1999) study included two different radio propagation models as discussed later in this chapter. We found that LQP algorithms used various input parameters with signal power and location measurements being the most popular. Older LQP algorithms 


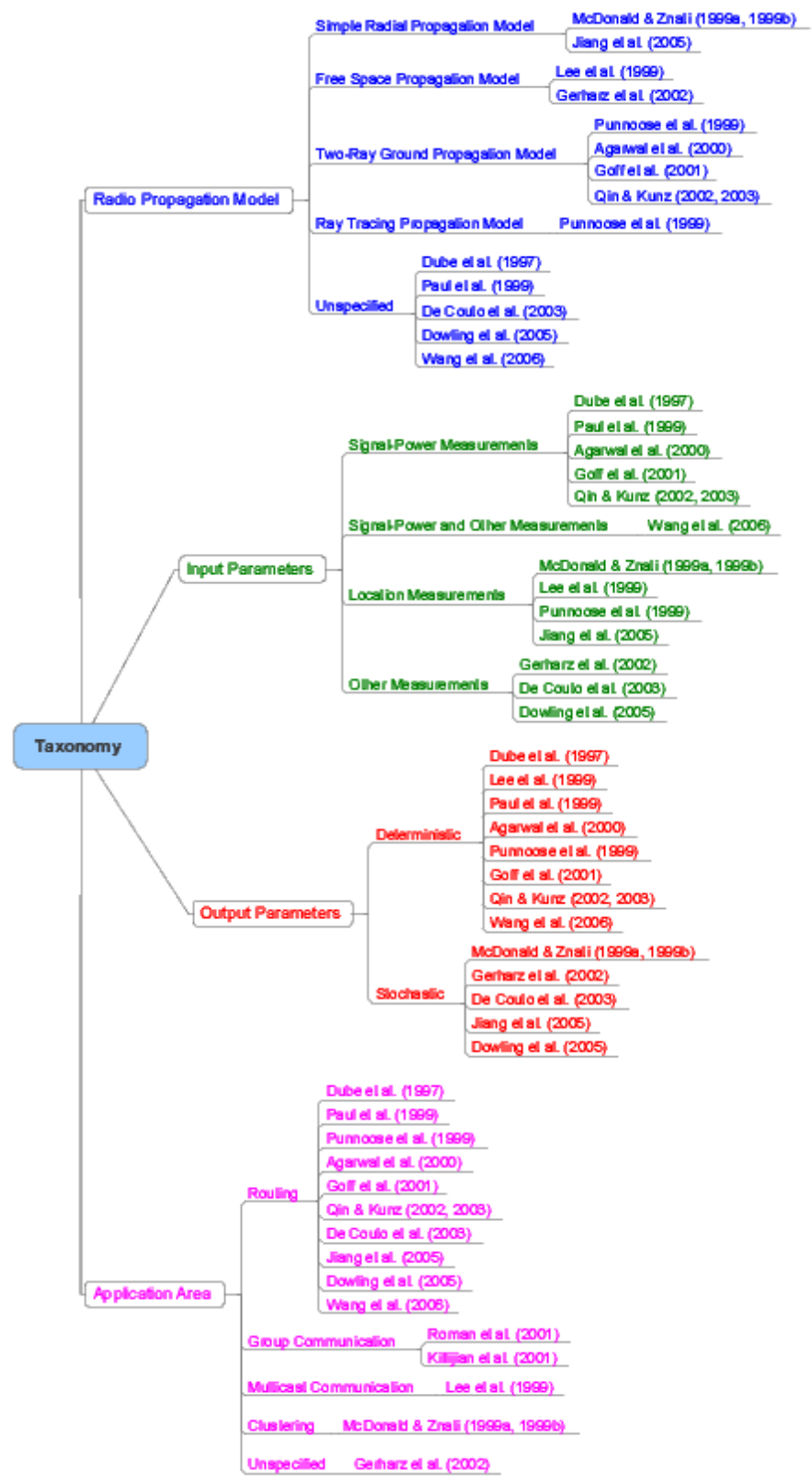

Fig. 1. Taxonomy of LQP algorithms by different criteria. The surveyed algorithms use a variety of input parameters. Older LQP algorithms tend to provide deterministic predictions while newer algorithms focus on stochastic predictions. Routing has been by far the most important application area for LQP. The underlying radio propagation model is unspecified for a considerable number of algorithms. 
tend to provide deterministic predictions while newer algorithms favour stochastic predictions. The most important application area for LQP in the literature was found in routing. For a considerable number of LQP algorithms the radio propagation model, on which these algorithms were based and evaluated, was unspecified.

All LQP algorithms are based on radio propagation models that characterize the propagation effect of path loss but do not account simultaneously for large-scale and wideband small-scale fading effects. However, the latter effects affect the radio propagation significantly (see, for example, Neskovic et al. 2000) and path-loss-only models do not consequently represent the real-world behavior well. To avoid the detailed repetition of this crucial critique, we refer to LQP algorithms based on such radio propagation models as LQP algorithms being based on 'path-loss-only' radio propagation models.

In this chapter, we refer to 'indirect' and 'direct' evaluations of LQP algorithms. We refer to an indirect evaluation if the accuracy of a LQP algorithm is evaluated by demonstrating that some metric, which refers to a higher-layer protocol, has changed due to the use of LQP. A higher-layer protocol might be a routing protocol or a multicast protocol and typical metrics are packet latency, packet delivery ratio and data throughput. In contrast, an evaluation is direct if the accuracy of a LQP algorithm is assessed by comparing its prediction of some quantity, such as the probability of receiving future transmitted packets, directly with future measurements of this quantity.

\section{Notation and nomenclature}

In order to unify the various notations used to describe LQP algorithms, we introduce the following notation inspired by Harvey (1993, p. 33). Let $t$ denote the variable that represents time and $\mathrm{T}$ represent the point in time at which a prediction is made. Then $\tau$ is the prediction horizon that represents the amount of time into the future for which this prediction is made. We call the interval $[\mathrm{T}, \mathrm{T}+\tau]$ the 'prediction interval' and the interval $\left[t_{c}, T\right]$ the 'history interval' where $t_{c} \leq T$ is some point in time. The data collected within the history interval is used at time $\mathrm{T}$ to predict a future value of a quantity at $\mathrm{T}+\tau$. The probability of a transmitted packet being successfully received is symbolized by the packet reception probability (PRP) $\Phi$ with range $\Phi \in[0,1]$. We denote a predictor for a quantity by a tilde over its symbol. A deterministic prediction of the PRP at time $\mathrm{T}$ for $\mathrm{T}+\tau$ is then defined as:

$$
\tilde{\Phi}_{\mathrm{T}+\tau \mid \mathrm{T}}= \begin{cases}0 & - \text { if the packet is predicted at time } \mathrm{T} \text { not to be received at time } \mathrm{T}+\tau \\ 1 & - \text { if the packet is predicted at time } \mathrm{T} \text { to be received at time } \mathrm{T}+\tau\end{cases}
$$

A stochastic PRP prediction is defined as the predicted probability that a packet will successfully be received at time $\mathrm{T}+\tau$ :

$$
\Phi_{\mathrm{T}+\tau \mid \mathrm{T}} \rightarrow\{0, \ldots, 1\} \in \mathbb{R}
$$

It is intended that, based on the result of the prediction that a decision be made, whether or not to send data. If the prediction warrants it then data would be sent immediately 
following time $\mathrm{T}$. Given that in MANETS the distances are relatively short it is envisaged that the transmit time is negligible by comparison with the time the link is predicted to be stable. It is also important to discuss the interpretation of the time variables in the context of continuous real-world processes and the sampling by the computer. The stochastic processes of propagation effects investigated in this chapter are continuous. However, computers measure quantities derived from these processes in a discrete fashion. Assume, for example, a continuous stochastic real-world process $\mathrm{X}$ that is sampled with a sampling interval of $\mathrm{T}$. Hence, the sample $x_{t}$ corresponds to the realization of this process at time $t$. Assume that this sample has been taken at $t=10: 45: 00$ hours and that the sampling interval $\mathrm{T}$ is 0.5 seconds. If we now refer to a sample that was taken at $\mathrm{x}_{\mathrm{t}+8}$ this sample was not taken at 10:45:08 hours but at 10:45:04 hours since $x_{t+8}$ corresponds in the discrete system to $t+8 \mathrm{~T}$, which is $10: 45: 00$ plus 8 times 0.5 seconds. In general, the discrete samples $x_{t+c}$ correspond to samples of the continuous system at the actual time $t+c \tau$ while in the sampled computer representation $t$ and $c$ are some indices with the meaning explained above.

There are several other quantities that may be used or predicted by LQP algorithms. The location of a mobile node is represented by ' $\mathrm{o}$ '. Knowledge of successive locations allows for the derivation of the velocity. The magnitude of the velocity vector (i.e. the speed) is denoted by $v$ and the direction by $\mathrm{v}^{\prime}$. The signal power of a received packet is denoted by $R$ in Watts and by $R(d B m)$ when measured in $d B m$; the signal envelope is symbolized by $r$.

\section{LQP algorithms based solely on signal-power measurements}

In this section we present LQP algorithms that rely solely on signal-power measurements to predict link quality.

\subsection{Dube, Rais, Wang \& Tripathi (1997)}

LQP in MANETs has been introduced in the context of routing by Dube et al. (1997). Their on-demand routing protocol uses two conditions for route selection that are based on signal-power measurements. These signal-power measurements are assumed to represent future link quality albeit that the prediction horizon is not specified. The first condition requires that signal-power measurements for each link on a route have higher exponential average power than a threshold. The second condition demands that the exponential average signal power for each link has been above its threshold for a specified period of time. The first condition is termed 'signal stability', the second one 'location stability'. Applying both conditions leads to the selection of routes that have longer remaining lifetimes and therefore require less route maintenance. This was confirmed by simulations in which this routing protocol is compared to a simple, imaginary routing protocol that chooses the shortest route without considering signalpower measurements.

The LQP algorithm used to select routes with higher remaining lifetimes is given by the following equation: 


$$
\tilde{\mathrm{R}}_{\mathrm{T}+1 \mid \mathrm{T}}=\mathrm{c}_{1} \tilde{\mathrm{R}}_{\mathrm{T}}+\left(1-\mathrm{c}_{1}\right) \mathrm{R}_{\mathrm{T}}
$$

where $c_{1}$ is an experimentally determined constant, $\tilde{R}_{T}$ is the previously predicted signal power for some link, and $R_{T}$ is the last signal power value measured. If $\tilde{R}_{T+1 \mid T}$ is higher than an experimentally determined threshold, the link between the transmitter and receiver of a packet is classified as being 'strong', otherwise as being 'weak'. Since this prediction has only two possible outcomes we classify it as being deterministic. Routes with only strong links are preferred over routes that contain weak links. The expectation is that a route consisting of only strong links has a higher remaining lifetime than a route that also contains weak links. Older routes of strong links are preferred to younger routes.

A few observations are noteworthy. Equation (3) is proposed without derivation or justification. The constant $c_{1}$ as well as all thresholds are chosen by experience. The performance evaluation is based on simulations with a radio propagation model that considers only the distance between nodes but is not further specified. Hence, we cannot assess the applicability of this LQP algorithm to real-world environments.

\subsection{Paul, Bandyopadhyay, Mukherjee \& Saha (1999)}

Paul et al. (1999) propose an affinity metric that measures the strength of connection between two nodes. Based on this affinity metric a routing protocol for low volume data transmission and another for high volume data transmission are presented. The affinity metric is a value proportional to the predicted remaining lifetime of a link and is intended to identify routes with higher lifetime than without using such a predictive measure.

Let $\Delta_{\mathrm{Avg}}\left(\mathrm{R}_{\mathrm{T}}(\mathrm{dBm}),\left[\mathrm{T}-\mathrm{c}_{1}, \mathrm{~T}\right]\right)$ be the average rate of change of the signal-power evaluated at time $T$. This average is obtained using the samples $R_{T}(d B m)$ over a preceding interval of $c_{1}$ seconds. The 'affinity' quantity $g(t)$ is now defined as:

$$
g(t)= \begin{cases}\text { high } & - \text { if } \Delta_{\text {Avg }}\left(R_{T}(d B m),\left[T-c_{1}, T\right]\right) \geq 0 \\ \frac{c_{2}-R_{T}(d B m)}{\Delta_{\mathrm{Avg}}\left(R_{T}(d B m),\left[T-c_{1}, T\right]\right)} & - \text { if } \Delta_{\text {Avg }}\left(R_{T}(d B m),\left[T-c_{1}, T\right]\right)<0\end{cases}
$$

where $c_{2}$ is the signal power at the maximum transmission range and $R_{T}(d B m)$ is the last signal-power sample. If the value of $\Delta_{\mathrm{Avg}}\left(\mathrm{R}_{\mathrm{T}}(\mathrm{dBm}),\left[\mathrm{T}-\mathrm{c}_{1}, \mathrm{~T}\right]\right)$ is positive, the affinity between two nodes is increasing, given the authors' assumptions, since the nodes are moving closer to each other. In this case the affinity is classified simply as being 'high' since no link failure is predicted for the future. Otherwise, the affinity is calculated periodically using the above formula. A deterministic link failure prediction can now be given by the following equation:

$$
\tilde{\Phi}_{\mathrm{T}+\tau \mid \mathrm{T}}=\left\{\begin{array}{lr}
0 & \text { if } \mathrm{c}_{3} \mathrm{~g}(\mathrm{~T}) \leq \mathrm{T}+\tau \\
1 & \text { otherwise }
\end{array}\right.
$$

where $c_{3}$ is an experimentally determined constant that represents the proportionality factor between the affinity metric and the remaining link lifetime. 
Paul et al. (1999) evaluated the accuracy of their proposed LQP algorithm indirectly by showing that the number of route breaks can be significantly reduced in a variety of different scenarios when the affinity metric is used as a predictive measure to select routes with expected high remaining lifetimes.

The authors did not motivate or derive their LQP algorithm. Therefore, we do not know what radio propagation model was used as the basis for its derivation and hence, we cannot judge its applicability to real-world environments. Nevertheless, the presented LQP algorithm is interesting since it could provide accurate predictions at least for path-loss-only radio propagation models such as the Two-Ray Ground Propagation Model and the Free Space Propagation Model. These path-loss models are linear if the path loss is expressed in decibels versus the logarithmic distance. Consequently, the linear equations (4) and (5) are appropriate for these models, albeit with a certain prediction error if the signal power of nodes with constant speed vectors is measured in linear time intervals.

\subsection{Agarwal, Ahuja, Singh \& Shorey (2000)}

Agarwal et al. (2000) built their Route-Lifetime Assessment Based Routing (RABR) protocol on Paul et al.'s (1999) LQP algorithm discussed in the previous section. Since their work utilizes the affinity metric without changes, we omit a discussion of it here. RABR introduces three new features compared to previous routing protocols. First, route selection for Transmission Control Protocol (TCP) traffic is extended to consider the throughput function of TCP as well as the affinity metric. Since the throughput of TCP traffic can be approximated by a function of the number of hops (Gerla et al. 1999), route selection is based on the product of the weakest link's affinity and the bandwidth of that route. Therefore, a route that has a lower predicted remaining lifetime but higher bandwidth can be more suited to a transmission than the route that has the longest predicted remaining lifetime but with much lower bandwidth. Secondly, the affinity metric is employed to increase the effectiveness of route cache maintenance. Route caching reduces the latency of route discovery in on-demand routing protocols since an intermediate rather than the destination node can answer the route discovery request from some source node, if a suitable route exists in its cache. However, stale routes in the cache can increase latency considerably. Hence, a timeout that equals the expected lifetime of the route is assigned to each route cache entry such that no stale entries exist if the LQP is accurate. Thirdly, the authors introduce a power saving scheme that reduces the transmission power using the affinity metric. However, only between $3 \%$ to $5 \%$ of power was saved in their simulations.

The effectiveness of Paul et al.'s (1999) LQP ALGORITHM was demonstrated in simulations with NS-2. For a wide range of scenarios the performance of the RABR protocol was compared to other common routing protocols (Agarwal et al. 2000). For medium to large average node speeds the RABR protocol was shown to outperform its competitors in terms of TCP throughput and ratio of delivered to sent packets. However, the suggested LQP algorithm and its evaluation are based on a path-loss-only radio propagation model.

\subsection{Goff, Abu-Ghazaleh, Phatak \& Kahvecioglu (2001)}

Goff et al. (2001) were the first to introduce the concept of proactive routing for on-demand routing protocols. On-demand routing protocols exhibit low traffic overhead since they only 
update active routes that have been used in the recent past. However, updates are only triggered after a link failure is detected in which case multiple retransmissions have been unsuccessful. Therefore, time passes while a new route is being discovered which results in high mean and variance of packet latency. Proactive routing, however, tries to minimize this latency by initiating route discoveries early enough to have discovered alternative routes by the time links fail. The efficiency of proactive route discovery relies on accurate LQP. If a link is falsely predicted to fail early, unnecessary route discoveries are initiated and a route with lower quality than currently used might be chosen. However, if a link failure is predicted too late or not predicted at all, the route discovery does not finish before the link fails; hence, packet latency is increased.

The authors rely on the concept of preemption for enabling proactive route discovery. A communication task (e.g. route discovery) is initiated preemptively such that it can be completed even if links between two nodes fail when these two nodes move apart at maximum speed. The preemptive region starts at the maximum distance at which two nodes can be separated such that if they move apart at maximum speed, the communication task can still be completed. The preemptive region ends at the maximum transmission radius. The preemptive threshold is the signal power that corresponds to the beginning of the preemptive region and this threshold is intended to determine whether a node has entered the preemptive region with respect to another node.

The proposed LQP ALGORITHM differs from previous ones in that it is more a protocol than a mathematical expression. The idea behind this approach is that a number $c_{1}$ of 'ping-pong' rounds are exchanged between two nodes if a packet is received whose signal power is below the preemptive threshold $c_{2}$. If the signal power of $c_{3}$ or more of these $c_{2}$ packets are below the threshold or these packets are lost, the link is predicted to fail. The 'ping-pong' rounds are intended to reduce falsely predicted link failures that are caused by temporary fades due to small-scale fading. The preemptive threshold is calculated such that if two nodes move away from each other at maximum speed, the link failure is predicted early enough to allow a route discovery to complete before the link fails. Therefore, the threshold $c_{2}$ corresponds to the signal power at the beginning of the preemptive region that represents the difference between the maximum transmission distance and the product of the maximum relative speed between two connected nodes and the prediction horizon. For their evaluation Goff et al. (2001) set the number of 'ping-pong' rounds $c_{1}$ and the threshold $c_{3}$ to three. The LQP algorithm was derived considering the Two-Ray Ground Propagation Model and small-scale fading.

Significant improvements due to proactive instead of on-demand routing were shown for the Dynamic Source Routing (DSR) (Johnson \& Maltz 1996) and the Ad hoc On-demand Distance Vector (AODV) (Perkins et al. 2001) protocol in an augmented version of the NS-2 simulator. The simulator was augmented with an error model that assigns either a good or bad state to a link. In order to approximate small-scale fading, the signal power was decreased by division by a uniformly distributed random number between 2 and 100 when a bad state was assigned to a link. The mean length of staying in a state was set to 20000 packets in the good state and to 2 packets in the bad state. It was shown for both routing protocols that the number of broken routes and the packet latency was significantly reduced for constant-bit-rate traffic due to proactive route discovery. A similar reduction in packet latency was also demonstrated for TCP traffic. 
This deterministic LQP algorithm relies critically on the assumption of bidirectional links since the 'ping-pong' mechanism to mitigate small-scale fading does not work otherwise. Furthermore, this algorithm only allows for LQP for one future point in time and not for a set of points (interval). For example, the algorithm can be used to predict link failure for 2 seconds ahead but not $0.5,1.0,1.5$ and 2 seconds ahead simultaneously. This property is based on the protocol's nature. Since the 'ping-pong' mechanism is triggered by the preemptive threshold that is a function of the prediction horizon, it cannot be trigged for a different threshold simultaneously. In terms of evaluation, the accuracy of the proposed approximation model for small-scale fading is questionable. Although small-scale fading affects the signal power of all transmitted packets, only deep fades, which occur very rarely with a probability of 1 in 10000 packets in their model, are simulated. Goff et al. (2001) do not explain how this probability is obtained. Furthermore, the important propagation effect of large-scale fading has not been considered. For these reasons, the applicability of the proposed LQP algorithm to real-world environments is unclear.

\subsection{Qin \& Kunz (2002) and (2003)}

Qin \& Kunz (2002) add proactive route discovery to the DSR protocol similarly to Goff et al.'s (2001) work. However, Qin \& Kunz (2002) use a different LQP ALGORITHM and they focus on reducing the number of dropped packets by means of LQP rather than improving the number of broken routes and the packet latency. Packets can be dropped during route discovery if no alternative route has yet been found. This loss of packets causes significant degradation in throughput as shown, for example, for TCP (Holland $\&$ Vaidya 2002). However, if alternative routes are discovered proactively before a link, and therefore a route, fails, no packets will be lost.

Qin \& Kunz (2002) derive their LQP ALGORITHM trigonometrically using the same principle as Lee et al. (1999) and Jiang et al. (2005) whose works are discussed later in this chapter. However, the latter two approaches use location measurements to predict the time of link failure, while this algorithm uses only signal-power measurements. These signalpower measurements are used to estimate the distance between two nodes by assuming that only the path loss as given by the Two-Ray Ground Propagation Model determines the signal power. The LQP ALGORITHM is derived from the Law of Cosines (Kay 2001, pp. 2731) under the assumption that nodes maintain their speed and direction during the history and prediction intervals. Therefore, the time of link failure $\tilde{\Phi}_{\mathrm{t}>\mathrm{T} \mid \mathrm{T}}$ is predicted at time $\mathrm{T}$ by:

$$
\tilde{\Phi}_{\mathrm{t}>\mathrm{T} \mid \mathrm{T}}=\frac{\sqrt{\mathrm{g}_{2}^{2}-4 \mathrm{~g}_{1} \mathrm{~g}_{3}}-\mathrm{g}_{2}}{2 \mathrm{~g}_{1}}
$$

where

$$
\begin{aligned}
& \mathrm{g}_{1}=\mathrm{t}_{\mathrm{T}-1} \mathrm{~g}_{4} \sqrt{\mathrm{R}_{\mathrm{T}-1} \mathrm{c}_{1}} \\
& \mathrm{~g}_{2}=\sqrt{\mathrm{c}_{1}}\left(\left(\sqrt{\mathrm{R}_{\mathrm{T}-2}}-\sqrt{\mathrm{R}_{\mathrm{T}-1}}\right)-\mathrm{t}_{\mathrm{T}-1}^{2} \mathrm{~g}_{4} \sqrt{\mathrm{R}_{\mathrm{T}-1}}\right) \\
& \mathrm{g}_{3}=\mathrm{t}_{\mathrm{T}-1} \sqrt{\mathrm{R}_{\mathrm{T}-1} \mathrm{c}_{1}}-\mathrm{t}_{\mathrm{T}-1} \sqrt{\mathrm{R}_{\mathrm{T}-2} \mathrm{R}_{\mathrm{T}-1}} \\
& \mathrm{~g}_{4}=\frac{\mathrm{t}_{\mathrm{T}-1} \sqrt{\mathrm{R}_{\mathrm{T}-2} \mathrm{R}_{\mathrm{T}-1}}+\mathrm{T} \sqrt{\mathrm{R}_{\mathrm{T}-1} \mathrm{R}_{\mathrm{T}}}-\mathrm{T} \sqrt{\mathrm{R}_{\mathrm{T}-2} \mathrm{R}_{\mathrm{T}}}-\mathrm{t}_{\mathrm{T}-1} \sqrt{\mathrm{R}_{\mathrm{T}-1} \mathrm{R}_{\mathrm{T}}}}{\sqrt{\mathrm{R}_{\mathrm{T}-1} \mathrm{R}_{\mathrm{T}}}\left(\mathrm{t}_{\mathrm{T}-1} \mathrm{~T}^{2}-\mathrm{Tt}_{\mathrm{T}-1}^{2}\right)}
\end{aligned}
$$


and $c_{1}$ symbolizes the signal-power threshold below which packets are lost, $R_{t}$ represents the signal power measured at time $t$, and $t_{T-x}$ denotes the times at which samples are taken. Equation (6) is only applied if three consecutive packets have decreasing signal power. Otherwise the nodes are assumed to be moving closer and the link is predicted not to fail. The deterministic PRP predictor is therefore defined as:

$$
\tilde{\Phi}_{\mathrm{T}+\tau \mid \mathrm{T}}=\left\{\begin{array}{cc}
0 & \text { if } \mathrm{T}+\tau \geq \tilde{\Phi}_{\mathrm{t}>\mathrm{T} \mid \mathrm{T}} \\
1 & \text { otherwise }
\end{array}\right.
$$

Qin \& Kunz (2002) also discuss how their LQP ALGORITHM can be made resilient against power fluctuations. They propose to preprocess the sampled signal power by linear regression and showed, for one example situation, that the accuracy of their link failure prediction increased compared to not using any preprocessing. This result stems from simulations in which the signal power varies by random noise of between $5 \%$ and $10 \%$ from its mean. However, there are a number of problems with this approach. First, Qin \& Kunz (2002) do not define the cause of the power variations. Do these variations refer to measurement noise or radio propagation effects? Secondly, the distribution and correlation structure of the noise process is not given. However, linear regression is only an unbiased estimator if the noise process is normally distributed and uncorrelated (Hines et al. 2002, pp. 409-414). These assumptions are invalid, for example, when the noise refers to small-scale and large-scale fading. Thirdly, the authors do not describe why the power varies only between the small values of $5 \%$ and $10 \%$ from its mean.

Qin \& Kunz's (2002) work is one of the few studies that directly evaluated the accuracy of the proposed LQP ALGORITHM. In a simulation with the NS-2 simulator it was shown that more than $90 \%$ of lost packets were successfully predicted for different mobility patterns. It was also demonstrated that the number of dropped packets in DSR due to unavailable routes was reduced by between $20 \%$ and $44.95 \%$ while the overhead of control messages increased by up to $33.5 \%$. This increase in control messages, however, did not cause network congestion or other significant, negative side effects. However, these simulations, as well as the LQP ALGORITHM itself, are based on a 'path-loss-only' radio propagation model and even the previously discussed simple power variations were omitted.

\section{LQP algorithms based on signal-power and other measurements}

In this section we describe algorithms that use signal power as well as other types of measurements to predict link quality. These other measurements can be, for example, the average packet loss ratio or the hop count.

\subsection{Wang, Martonosi \& Peh (2006)}

Wang et al. (2006) suggest the use of supervised learning to predict the quality of links in static wireless sensor networks. The term 'prediction' refers in this context more to estimation or classification of current link quality than to the forecast of link quality for a specific time into the future. Nevertheless, since Wang et al. (2006) assume similar to De Couto et al. (2003) and Dowling et al. (2005) justifiably that the current link quality represents the future link quality to some degree, we include this work in our survey. The 
proposed link quality estimator replaces the original link quality metric ETX in the MintRoute (Woo et al. 2003) routing protocol and its impact on different higher-layer metrics (e.g. packet delivery ratio and packet latency) in the original and augmented MintRoute protocol is assessed. The suggested link quality estimator intends to be more accurate in scenarios with high network traffic. In these situations snooping-based methods such as ETX can underestimate link quality due to high packet loss of probe packets that is caused by the hidden and exposed terminal problems as well as the unfairness of the 802.11 medium access control (MAC) protocol (Tobagi \& Kleinrock 1975, Xu \& Saadawi 2001).

Supervised learning establishes the mapping between an input and an output measure in an offline training phase. The input measure consists of a feature vector that contains features that can be measured by the system under consideration. These features are selected by humans at design time and they should have a meaningful relation to the desired output measure. If the output measure has a finite set of outcomes, the problem is called classification. In the training phase, the learning algorithm tries to minimize the classification error between the input measure and the given output measure. The output measure in Wang et al.'s (2006) work is the link state, which is assigned to be one of the three states 'bad', 'medium' and 'good'. Different metrics are suggested as the feature vector:

- The signal power of received packets.

- The usage of send and forward buffers to detect network congestion.

- The hop count of the sensor node from the base station.

- The average packet loss ratio, which is determined by a snooping-based method such as ETX, for the link in both directions.

Interestingly, the classification accuracy using all features was $80.8 \%$ and still around $70 \%$ for using the signal power or the average packet-loss ratio alone. Therefore, the classification was only moderately improved by using additional features.

Packet delivery ratio, packet latency and a fairness index were the metrics chosen to compare the performance of the original MintRoute protocol with its augmented version. The fairness index is a summary statistic that captures the packet delivery ratio for all nodes of a network. It has a value between 0 and 1 and is 1 when all nodes have the same packet delivery ratio. The evaluation was conducted in a test bed of 30 wireless sensors nodes that were located across multiple offices in a building. The wireless nodes communicated over 802.15.4 (ZigBee) and the packet size for all experiments was set to 29 bytes. All nodes were static and the link quality in the network varied due to changes in the environment, radio propagation effects and different network loads. The new LQP ALGORITHM led to significantly higher packet delivery ratios, a comparable average packet latency and improved fairness of the MintRoute protocol.

The proposed supervised learning approach was shown to be effective for wireless networks with static nodes only. However, it is not clear if this method is also applicable to MANETs with node mobility. Moreover, the suggested LQP ALGORITHM is suitable for selecting routes in routing protocols leading to the reported performance improvements but is not able to predict link quality for a specifiable prediction horizon. Furthermore, all results were obtained for a small packet size. Since in many application scenarios longer and varying packet sizes can be expected, it is uncertain whether this learning approach will extend to these situations since the packet loss depends on the packet length, as it was, for example, observed in the work of De Couto et al. (2003). 


\section{LQP algorithms based solely on location measurements}

In this section we present LQP algorithms that rely solely on location measurements for their predictions. Location measurements are usually obtained by GPS in outdoor environments. However, GPS has a user-equivalent range error of $19.2 \mathrm{~m}$ (Grewal et al. 2001, pp. 128-130) and is therefore fairly inaccurate. The user-equivalent range error summarizes the overall error experienced by a user from the various error sources of the GPS (e.g. transmission delays caused by the atmosphere, inaccuracy of satellite clocks, etc.). Furthermore, GPS requires a line-of-sight (LOS) connection to at least three satellites that are often not in view in dense urban environments.

\subsection{Lee, Su \& Gerla (1999)}

Lee et al. (1999) presented a deterministic PRP predictor to enhance the performance of the On-Demand Multicast Routing Protocol (ODMRP) from Gerla et al. (1998). While the original ODMRP protocol uses a fixed refresh interval for periodic flooding to update each nodes' knowledge about the network topology, in the augmented ODMRP protocol the refresh interval is set to some time before the link with the shortest predicted remaining lifetime is assumed to fail. This anticipation of topology changes reduces latency by updating the network topology before it actually changes. LQP is also employed to select routes with longer remaining lifetimes such that route maintenance is reduced.

Lee et al.'s (1999) predictor is based on location measurements, the resulting speed vector and the Free Space Propagation Model. Although the authors do not give any mathematical derivation of their predictor, it is obvious from the expression that it is derived by trigonometry. If two nodes keep the same speed and direction within the prediction interval, the time until the link fails corresponds to the time at which the nodes are predicted to be further apart than the maximum transmission radius. Formally, let the coordinates of the mobile node at time $T$ be $\left(x_{i}, y_{i}\right)$. Let $v_{i}$ be its speed and $v_{i}^{\prime}$ its direction. Then the link failure time $\tilde{\Phi}_{\mathrm{t}>\mathrm{T} \mid \mathrm{T}}$ for the link between two nodes $i$ and $\mathrm{j}$ is predicted by:

$$
\tilde{\Phi}_{\mathrm{t}>\mathrm{T} \mid \mathrm{T}}=\frac{-\left(\mathrm{g}_{1} \mathrm{~g}_{2}+\mathrm{g}_{3} \mathrm{~g}_{4}\right)+\sqrt{\mathrm{c}_{1}^{2}\left(\mathrm{~g}_{1}^{2}+\mathrm{g}_{3}^{2}\right)-\left(\mathrm{g}_{1} \mathrm{~g}_{4}-\mathrm{g}_{2} \mathrm{~g}_{3}\right)^{2}}}{\mathrm{~g}_{1}^{2}+\mathrm{g}_{3}^{2}}
$$

Where:

$$
\begin{aligned}
& \mathrm{g}_{1}=\mathrm{v}_{\mathrm{i}} \cos \left(\mathrm{v}_{\mathrm{i}}^{\prime}\right)-\mathrm{v}_{\mathrm{j}} \cos \left(\mathrm{v}_{\mathrm{j}}^{\prime}\right) \\
& \mathrm{g}_{2}=\mathrm{x}_{\mathrm{i}}-\mathrm{x}_{\mathrm{j}} \\
& \mathrm{g}_{3}=\mathrm{v}_{\mathrm{i}} \sin \left(\mathrm{v}_{\mathrm{i}}^{\prime}\right)-\mathrm{v}_{\mathrm{j}} \sin \left(\mathrm{v}_{\mathrm{j}}^{\prime}\right) \\
& \mathrm{g}_{4}=\mathrm{y}_{\mathrm{i}}-\mathrm{y}_{\mathrm{j}}
\end{aligned}
$$

and $c_{1}$ is the maximum transmission radius. If $v_{i}=v_{j}$ and $v_{i}^{\prime}=v_{j}^{\prime}$ then the link failure time is set to infinity without applying (12) since the nodes are maintaining a constant distance. The deterministic PRP predictor is now defined by: 


$$
\tilde{\Phi}_{\mathrm{T}+\tau \mid \mathrm{T}}=\left\{\begin{array}{cc}
0 & \text { if } \mathrm{T}+\tau \geq \tilde{\Phi}_{\mathrm{t}>\mathrm{T} \mid \mathrm{T}} \\
1 & \text { otherwise }
\end{array}\right.
$$

The accuracy of the proposed LQP ALGORITHM was shown indirectly in the GloMoSim simulator (Bajaj et al. 1999) by comparing different aspects of the enhanced multicast protocol with the original one. It was shown that the packet delivery ratio of the enhanced ODMRP protocol was considerably higher than the original protocol, especially for high node speeds. Moreover, the packet latency was reduced significantly by anticipation of topology changes. However, the suggested LQP ALGORITHM and its evaluation are based on a 'path-loss-only' radio propagation model.

\subsection{McDonald \& Znati (1999b) and (1999a)}

McDonald \& Znati (1999b) suggest a stochastic PRP predictor for a cluster-based routing framework (McDonald \& Znati 1999a). The idea behind this routing approach is to divide the mobile network into clusters that guarantee with certain probability for a certain length of time unbroken links for nodes within this cluster. The probability and length of time are specifiable. Table-driven routing is employed within a cluster; on-demand routing is used for inter-cluster routing. Table-driven routing protocols periodically update the routing information throughout the network for all possible routes. Therefore, for highly dynamic topologies the overhead is high while latency is still low since routes have been precomputed. On-demand routing protocols in contrast only maintain previously used routes. Hence, the traffic overhead is low but latency is high if a route between source and destination has to be discovered. Mixing these different routing schemes aims to balance the trade-off between table-driven and on-demand routing based on clustering nodes. The efficiency of this clustering relies on low effort for cluster maintenance that is achieved by LQP.

McDonald \& Znati (1999b) derive their LQP ALGORITHM from their proposed mobility model and the simple Radial Propagation Model. Their mobility model assumes that each node's movements consist of a sequence of random length intervals called mobility epochs during which it moves in a constant direction at constant speed. The parameters of this mobility model are termed the 'mobility profile'. Formally, for a node i the epoch length is assumed to be an exponential Independent and Identically Distributed (IID) random variable with mean $1 / c_{1}$. The speed $v_{i}$ is taken to be an IID random variable with mean $c_{2}$ and variance $c_{3}$. The direction of movement is also a uniformly distributed IID random variable having a value of between 0 and 360 degrees. This mobility model is similar to the Random Walk Mobility Model (Polya 1921) and McDonald \& Znati (1999b) do not explain how their model relates to the commonly used Random Waypoint Mobility Model (Johnson \& Maltz 1996) or any other mobility model. The predicted PRP for a link $(i, j)$ between two nodes is given by:

$$
\begin{aligned}
& \tilde{\Phi}_{\mathrm{T}+\tau \mid \mathrm{T}} \approx \frac{1}{2}\left(1-\mathrm{I}_{0}\left(\frac{-2 \mathrm{c}_{4}^{2}}{\mathrm{~g}(\mathrm{~T}+\tau)}\right) \exp \left(\frac{-2 \mathrm{c}_{4}^{2}}{\mathrm{~g}(\mathrm{~T}+\tau)}\right)\right) \\
& \mathrm{g}(\mathrm{t})=2 \mathrm{t}\left(\frac{\mathrm{c}_{3, \mathrm{i}}^{2}+\mathrm{c}_{2, \mathrm{i}}^{2}}{\mathrm{c}_{1, \mathrm{i}}}+\frac{\mathrm{c}_{3, \mathrm{j}}^{2}+\mathrm{c}_{2, \mathrm{j}}^{2}}{\mathrm{c}_{1, \mathrm{j}}}\right)
\end{aligned}
$$


where $I_{0}$ is the modified Bessel function of the first kind and zero order, and $c_{4}$ is the maximum transmission radius. As evident from (19) the PRP is predicted based on the mobility profile $\left(c_{1}, c_{2}, c_{3}\right)$. However, McDonald \& Znati (1999a) assume these values to be known by each node. Clearly, this assumption is valid for simulations only. Nevertheless, real-world use can be enabled when mobility profiles are estimated by each node using location measurements. We therefore classify this algorithm as being based on location measurements.

McDonald \& Znati (1999b) evaluated the accuracy of their LQP ALGORITHM in a custombuilt, discrete event simulator. On average, the LQP was accurate for longer prediction horizons but substantially underestimated link quality for shorter horizons as also observed by Jiang et al. (2005). The accuracy of the suggested LQP ALGORITHM was also assessed indirectly in McDonald \& Znati (1999a) where it was shown that a proposed clustering algorithm, which uses (18) and (19), led to clusters that were stable and adaptive to node mobility. Again, these evaluation results and the design of the authors' LQP ALGORITHM stem from a 'path-loss-only' radio propagation model.

\subsection{Punnoose, Nikitin, Broch \& Stancil (1999)}

Punnoose et al. (1999) propose a signal-power predictor for MANETs. Their approach uses location measurements from which the signal power is predicted by using a site-specific three-dimensional ray tracing propagation model with terrain map. The following components are suggested for their model:

- Non-location-based prediction. In the absence of location information this component predicts signal power by extrapolation of past signal-power samples.

- Mobility model. Probable future node locations are predicted using past location and speed measurements.

- Terrain map. The terrain map is used to provide the radio propagation model with necessary data about the environment and is updated when the environment changes.

- Radio propagation and communication model. This model predicts link quality by considering the predicted node locations, the terrain map and feedback about the accuracy of past predictions.

- Decision maker. The decision maker interacts with the network protocol to determine which links should be tracked and to provide LQP for the network.

While the above components have been suggested as a general architecture, these were only partly implemented and evaluated in the work of Punnoose et al. (1999). The non-locationbased prediction was, for example, neither explained nor implemented. A terrain map was used for one evaluation scenario; however, it was not employed for a second scenario. The update of the terrain map was omitted. The mobility model for prediction was implemented and past information was used to increase prediction accuracy for cyclic mobility patterns. Nevertheless, the authors give no details about their algorithm for mobility prediction such that we cannot assess this component. They used two different radio propagation models in their work. In one scenario, a three-dimensional "N+2 ray+diffraction" propagation model (Punnoose et al. 1999) was employed that takes into account a direct ray, a ground reflected ray and rays reflected off $\mathrm{N}$ objects as well as diffraction from these objects. This 
propagation model relies on terrain maps that contain the shapes of buildings with reflection and diffraction coefficients of their materials. In order to reduce the computational cost of this ray tracing model, the search for all multipath sources was restricted. Specific details about this proposed enhancement such as the resulting computational effort and the reduction in accuracy were not given by Punnoose et al. (1999). In a second evaluation scenario the Two-Ray Ground Propagation Model with empirically determined, site-specific constants was employed for prediction. No explanation was given as to why two different radio propagation models have been chosen for the two scenarios. Furthermore, the suggested use of past locations was omitted from the prediction model implemented. The decision maker tracked the link quality for all nodes and interacted with the DSR protocol such that routes with higher predicted link quality were preferred.

The LQP accuracy was evaluated within an experimental outdoor test bed. In the first scenario, five mobile nodes in vehicles followed a looped course. Each mobile node was equipped with a differential GPS receiver. For a link between two nodes the predicted and measured signal power was displayed together with the packet loss as three separate graphs in Punnoose et al.'s (1999) paper. Unfortunately, these graphs use a large time scale that precludes a direct evaluation of the prediction accuracy in the order of seconds. Furthermore, the prediction horizon was not given such that the span of the prediction into the future is unknown. In the second scenario, one mobile node and two stationary nodes were used to show that the augmented DSR protocol chose routes with higher link quality due to the proposed LQP ALGORITHM. In summary, the results presented were not sufficiently detailed to allow us a full assessment.

\subsection{Jiang, He \& Rao (2005)}

Jiang et al. (2005) present a stochastic LQP ALGORITHM that has some similarity to McDonald \& Znati's (1999b) approach. Both are based on the prediction of node mobility for exponential mobility models such as the Random Walk Mobility Model. Furthermore, both assume a simple Radial Propagation Model such that two nodes are connected if they are within a specified maximum transmission radius. Both approaches predict link quality to assess how reliable the connection between nodes is. While McDonald \& Znati (1999b) use this information for clustering, Jiang et al. (2005) employ it for selection of routes in the context of on-demand routing. One major difference between both LQP approaches is that McDonald \& Znati's (1999 $\underline{b}$ ) LQP ALGORITHM predicts at time T the PRP for T $+\tau$, while Jiang et al.'s (2005) approach predicts the probability that all packets, which will be sent between $\mathrm{T}$ and $\mathrm{T}+\tau$, are received. However, this latter definition of link failure, which requires for an available link that every sent packet will be received, is restrictive. A link might experience brief periods of unavailability due to temporary packet loss, which is caused by small-scale fading, but is available for all times outside this brief period. Another considerable difference between both approaches is that for a link McDonald \& Znati's (1999 b) LQP ALGORITHM predicts the PRP probability for a specifiable time $\mathrm{T}+\tau$ while Jiang et al.'s (2005) LQP ALGORITHM predicts first the link failure time and subsequently estimates the probability that the link will really last until the predicted link failure time.

More specifically, Jiang et al.'s (2005) LQP ALGORITHM works in the following two stages. First, a measurement-based approach predicts the time of link failure. The underlying 
assumption is that nodes will keep their past speed and direction within the prediction interval. Secondly, based on this link failure time the probability that the link will be available through the whole interval is calculated. For this prediction the situations are considered in which the speed and direction of the nodes do not change and in which one node or both nodes change their direction and speed.

The time until link failure $\tilde{\Phi}_{\mathrm{t}>\mathrm{T} \mid \mathrm{T}}$ for two nodes is derived similarly to Lee et al. (1999) and Qin \& Kunz (2002) by employing trigonometry. Therefore, $\tilde{\Phi}_{\mathrm{t}>\mathrm{T} \mid \mathrm{T}}$ is predicted at time T by:

$$
\tilde{\Phi}_{\mathrm{t}>\mathrm{T} \mid \mathrm{T}}=\frac{\sqrt{\mathrm{g}_{2}^{2}+4 \mathrm{c}_{1}^{2}-4 \mathrm{~g}_{1} \mathrm{~g}_{3}}-\mathrm{g}_{1}}{2 \mathrm{~g}_{3}}-\mathrm{T}
$$

where

$$
\begin{aligned}
& \mathrm{g}_{1}=\frac{\mathrm{d}_{\mathrm{T}-1}^{2} \mathrm{~T}-\mathrm{d}_{\mathrm{T}}^{2} \mathrm{t}_{\mathrm{T}-1}-\mathrm{d}_{\mathrm{T}-2}^{2}\left(\mathrm{~T}-\mathrm{t}_{\mathrm{T}-1}\right)}{\mathrm{t}_{\mathrm{T}-1} \mathrm{~T}\left(\mathrm{t}_{\mathrm{T}-1}-\mathrm{T}\right)} \\
& \mathrm{g}_{2}=\frac{\mathrm{d}_{\mathrm{T}-1}^{2} \mathrm{~T}-\mathrm{d}_{\mathrm{T}}^{2} \mathrm{t}_{\mathrm{T}-1}-\mathrm{d}_{\mathrm{T}-2}^{2}\left(\mathrm{~T}^{2}-\mathrm{t}_{\mathrm{T}-1}^{2}\right)}{\mathrm{t}_{\mathrm{T}-1} \mathrm{~T}\left(\mathrm{t}_{\mathrm{T}-1}-\mathrm{T}\right)} \\
& \mathrm{g}_{3}=\mathrm{d}_{\mathrm{T}-2}^{2}
\end{aligned}
$$

and $c_{1}$ denotes the maximum transmission range, $d_{t}$ the distance between two nodes measured at time $t . t_{T-x}$ symbolizes the times at which samples are taken.

Jiang et al. (2005) stated that the distance could either be determined by location measurements or be approximated by signal-power measurements. However, since Jiang et al. (2005) used location measurements for their evaluation and they did not elaborate on how signal-power measurements can be employed, we classify this predictor as being based on location measurements. Note that Qin \& Kunz (2002) proposed a very similar predictor that corresponds to Jiang et al.'s (2005) predictor with the distance being estimated by signal-power measurements using the Two-Ray Ground Propagation Model.

Let $\mathrm{g}\left(\tilde{\Phi}_{\mathrm{t}>\mathrm{T} \mid \mathrm{T}}\right)$ denote the predicted probability at time $\mathrm{T}$ that all future packets will be received successfully until the link is predicted to fail at $\tilde{\Phi}_{t>T \mid T}$. Considering that the nodes might change their speed and direction, this probability is now predicted by:

$$
\mathrm{g}\left(\tilde{\Phi}_{\mathrm{t}>\mathrm{T} \mid \mathrm{T}}\right) \approx \frac{1-\mathrm{e}^{-2 \mathrm{c}_{2} \tilde{\Phi}_{\mathrm{t}>T \mathrm{~T}}}}{2 \mathrm{c}_{2} \tilde{\Phi}_{\mathrm{t}>\mathrm{T} \mid \mathrm{T}}+\mathrm{c}_{3}}+\frac{2 \mathrm{c}_{2} \tilde{\Phi}_{\mathrm{t}>\mathrm{T} \mid \mathrm{T}} \mathrm{e}^{-2 \mathrm{c}_{2} \tilde{\Phi}_{\mathrm{t}>\mathrm{T} \mid \mathrm{T}}}}{2}
$$

where $1 / c_{2}$ is the mean epoch length of the mobility model and $c_{3}$ is a constant that is updated at runtime. The mean epoch length is the amount of time in which, on average, the nodes do not change speed or direction and its value is dependent on the mobility model. In order to reduce the computational cost the predictor (24) is an approximation of the exact solution to the problem as defined by Jiang et al. (2005). More specifically, the predictor only considers changes of the nodes' speed and direction that lead to nodes moving further away 
but does not consider changes that lead to higher link quality as in the case of nodes moving closer. Consequently, the constant $c_{3}$ was introduced to correct for the difference between the approximate and exact solutions by comparing the predicted with the actual probability of link failure at run-time of the algorithm. Since the determination of $c_{3}$ is complex, we refer the reader to Jiang et al.'s (2005) paper for further details.

The LQP ALGORITHM was evaluated in the OPNET (MIL 3 Inc. 2006) simulator. It was shown that (24) is, on average, a good predictor for remaining lifetime of links for exponential mobility models such as the Random Walk Mobility Model. However, the accuracy was significantly reduced for non-exponential mobility models such as the Random Waypoint Mobility Model. Furthermore, we noticed that the scale of the presented graphs emphasized long-term prediction horizons such that we cannot comment on the prediction accuracy for short-term horizons of the order of a few seconds. Jiang et al. (2005) also showed that packet latency, packet loss ratio and the ratio of received to delivered packets were all significantly improved for the DSR protocol when routes with longer remaining lifetimes were selected based on their LQP ALGORITHM. However, this improvement could only be observed for exponential mobility models and the improvement was only minor for the non-exponential Random Waypoint Mobility Model. All results and the proposed LQP ALGORITHM were derived by a 'path-loss-only' radio propagation model.

\section{LQP algorithms based on other measurements}

In this section we introduce LQP algorithms that use measurements other than signal power and location for their predictions. Possible measurement metrics are the link age or metrics that calculate ratios based on the number of transmitted and received packets in the recent past.

\subsection{Gerharz, Waal, Frank \& Martini (2002)}

Gerharz et al. (2002) present two metrics that are intended to select the link with the highest remaining lifetime from a set of links. Both metrics are based on measurements of link age and its distribution. The first metric recommends choosing the link with the highest predicted average remaining lifetime. The second metric advocates choosing the link with the highest time at which this link is predicted to fail with $25 \%$ probability. Both metrics led in simulations to link choices that result in links with higher remaining lifetimes than choosing links randomly. For most scenarios examined these metrics also led to links with higher remaining lifetimes than choosing the oldest link.

The proposed metrics for LQP are not intended to accurately predict the remaining lifetime of links; rather they help to select links that are likely to have the longest remaining life. Gerharz et al. (2002) derived their metrics empirically from the link age data that was obtained by conducting a number of simulations for different mobility models. All simulations, including those for evaluation, were done with the Free Space Propagation Model and a maximum transmission radius of $50 \mathrm{~m}$. Therefore, the applicability of the proposed metrics is bound to this specific radio propagation environment and it is unclear if the metrics extend to more realistic radio propagation models or even the Free Space 
Propagation Model with a different maximum transmission radius. Furthermore, it was suggested that 30 minutes of link age data are required to use the proposed metrics. It was not explained how LQP can be provided within this initialization interval, however. Moreover, Gerharz et al. (2002) assumed that the mobility patterns (e.g. the parameters of the mobility model) are static with respect to time. Due to all these severe shortcomings it is unclear whether Gerharz et al.'s (2002) method can successfully be applied in more realistic simulations or real-world deployments.

\subsection{De Couto, Aguayo, Bicket \& Morris (2003)}

De Couto et al. (2003) introduce ETX in order to find higher-throughput routes in multi-hop wireless networks than those given by the traditional minimum hop-count metric, which is the most common metric used in ad hoc routing protocols. The Expected Transmission Count (ETX) metric is the expected number of transmissions, including possible retransmissions used in successfully making a unicast. It is assumed that the expected number of future transmissions corresponds to the estimated number of past transmissions. This estimation is realized by counting the number of transmitted and received packets with a subsequent calculation of their ratio. Metrics such as ETX, which use such counting mechanisms, are classified as being snooping-based.

The ETX metric was motivated by observations of link behavior in a wireless test bed of 29 nodes that were statically located on different floors in an office building. It was observed that the minimum hop-count metric often chooses routes with lower throughput than the optimal route. Furthermore, a high variance in packet reception ratios was perceived and around $20 \%$ of all links were asymmetric such that forward and reverse packet reception ratios differed by at least $25 \%$. However, it should be noticed that a high degree of asymmetry was observed in an experiment in which all nodes had broadcast packets for 5 seconds consecutively. Therefore, the packet reception ratios of all possible node pairs were determined over a large time span (the worst case is a difference of 140 seconds), such that the degree of asymmetry could be overestimated due to a change of propagation characteristics in the environment during that time span. These observations led to the following definition of the ETX metric $g_{e}$ for a link:

$$
\mathrm{g}_{\mathrm{e}}=\frac{1}{\mathrm{~g}_{\mathrm{f}} \mathrm{g}_{\mathrm{r}}}
$$

where $g_{f}$ and $g_{r}$ are the measured packet reception ratios for the forward and reverse links respectively. The ETX metric for a route is simply the sum of the ETX metrics of the links that constitute the route. The proposed metric has several desirable properties:

- ETX is based on packet reception ratios that are strongly correlated to the throughput.

- ETX considers the asymmetry of links by incorporating the packet loss in both directions.

- $\quad$ ETX penalizes routes with higher hop counts that have naturally a lower throughput.

The snooping interval was set to 10 seconds for all trials. However, an explanation of this value is missing. 
De Couto et al. (2003) confirmed the suitability of the ETX metric for routing in their wireless test bed of static nodes. It was demonstrated that the ETX metric leads to route selections with significantly higher throughput than the minimum hop-count metric in the DSDV protocol (Perkins \& Bhagwat 1994). Furthermore, it was observed that the throughput of the DSR protocol was only slightly improved by employing the ETX metric since DSR already includes link-layer transmission feedback and is therefore able to avoid links with high asymmetry. Moreover, the packet reception ratio was shown to depend on packet length. Since ETX determines this ratio via probe packets that are significantly longer than acknowledgements, it underestimates the reception probability for these acknowledgements, which leads to a biased estimation of the ETX metric as observed by the authors.

The ETX metric was evaluated in a static ad hoc network. Therefore, it is unclear how the above results translate to scenarios with node mobility, especially since large-scale and small-scale fading are spatial phenomena. Furthermore, the estimation of the ETX metric is representative for one packet length only since an influence of the packet length on the packet reception ratio was shown. Moreover, it is unclear how the ETX metric's performance, which was evaluated indoors, relates to the performance in an outdoor MANET. These LQP ALGORITHMs and the ETX metric are complementary concepts in which ETX is a higher-level metric.

\subsection{Dowling, Curran, Cunningham \& Cahill (2005)}

Dowling et al. (2005) present a framework for collaborative reinforcement learning to solve optimization problems in dynamic, distributed networks. As an example application for this framework, a routing protocol termed 'SAMPLE', which exploits online learning to minimize the cost of routing in MANETs, is proposed and evaluated. The learning goals are to maximize the overall network throughput, to minimize the ratio of delivered to undelivered packets and to minimize the number of transmissions for every packet sent.

Dowling et al. (2005) advocate a stochastic state transition model for links to model the cost of routes. This model predicts the probability of a successful unicast and is therefore a snooping-based method considering unicast packets as is the ETX metric of De Couto et al. (2003). The number of required transmissions for a successful unicast is given by:

$$
E\left[\frac{g_{s}}{g_{a}}\right]=\frac{g_{s}+c_{1} c_{2}\left(g_{r}+g_{b}+g_{p}\right)}{g_{a}+c_{2}\left(g_{r}+g_{b}+g_{p}\right)}
$$

where $g_{s}$ is the number of successful unicast transmissions, $g_{a}$ is the number of attempted unicast transmissions, $g_{r}$ is the number of received unicast transmissions, $g_{b}$ is the number of received broadcast transmissions, $g_{p}$ is the number of promiscuously received (overheard) unicast transmissions, and $c_{1}$ and $c_{2}$ are static constants. $c_{1}$ represents the belief in the successful transmission of a packet considering only received packets. $\mathrm{c}_{2}$ controls the weight between received and transmitted packets. Equation (2.26) is based on snooping transmitted and received packets within a fixed time interval that was set by Dowling et al. (2005) to 10 seconds. $c_{1}$ and $c_{2}$ were set to 0.5 and 0.2 respectively. 
The performance of the SAMPLE routing protocol was evaluated against AODV and DSR in the NS-2 simulator. Two scenarios were chosen with the first one representing the design assumptions of AODV and DSR in that a true ad hoc network without any fixed infrastructure was available. In the second scenario, these design assumptions were violated by including a subset of static nodes that provided a backbone. In the first scenario the packet delivery ratio of all routing protocols was high. However, when the NS-2 simulator was augmented such that links experienced random packet loss (without further details given about its statistical description), the SAMPLE protocol showed much better adaptivity. For packet loss of up to $20 \%$, SAMPLE showed packet delivery ratios above $85 \%$ while AODV and DSR exhibited only $60 \%$ and $10 \%$ respectively. In the second scenario it was shown that SAMPLE outperformed its competitors in terms of throughput and packet delivery ratio since it better utilized the backbone. While the accuracy of the proposed LQP ALGORITHM was confirmed indirectly by these simulations, it is unclear how the obtained results relate to real-world radio links since the quality of links is not arbitrarily random but a complicated stochastic process that is governed by diverse propagation effects depending on the environment. Furthermore, the constants in (26) and the observation length were set to values without any further explanation.

\section{Related literature}

In this section we discuss literature that does not suggest new LQP algorithms for MANETs but is related to LQP and its applications.

\subsection{Roman, Huang \& Hazemi (2001)}

Roman et al. (2001) use the concept of announced disconnections to create a group communication system for MANETs that provides consistency. Consistency is defined such that all messages are sent and delivered in the same group view by all members and no message is lost under the assumption that no node fails and communication failures are announced in advance. These properties simplify the development of mobile applications greatly since even frequent changes in the network topology are masked from the application layer by the principle of transparency. The announcement of disconnection relies on LQP that is provided by Roman et al. (2001) by means of the principle of safe distance. This safe distance is defined as the maximum distance at which one can guarantee that any communication task between two nodes can still be completed before the link between them fails, even if the nodes move away from each other at maximum speed. This concept corresponds to the preemptive region from Goff et al. (2001) with the difference that Goff et al.'s (2001) communication task is the discovery of routes, while Roman et al.'s (2001) task is the delivery of messages to achieve consistency. The proposed group communication system consists of a group discovery and a reconfiguration protocol. The former uses location information to determine if new groups or group members are in the vicinity such that either groups or members join. The latter protocol merges and splits groups, handles joins and leaves of group members and ensures consistency of message delivery. Reconfigurations rely on the principle that all members of a group are within a safe distance such that consistency can be provided due to announcement of disconnections. If one or more group members move outside the safe distance with respect to other members, the group is split into multiple groups. 
The work of Roman et al. (2001) focuses on the algorithms in their group communication system and does not provide any detailed LQP ALGORITHM. All the algorithms presented are only briefly outlined and no evidence of an implemented system is provided. Therefore, this work is of conceptual nature only. While we believe that LQP algorithms can be of great benefit for efficient group communication in MANETs, the practical feasibility of the proposed approach is questionable. Roman et al. (2001) assume perfect LQP as a basis for providing consistency. In this way, Fischer et al.'s (1985) impossibility results for achieving consensus in an asynchronous distributed system, which experiences arbitrary link failures, is circumvented and the complicated task of achieving consensus in MANETs with restricted link failure patterns is greatly simplified. However, the assumption of perfect LQP is unrealistic and thus should not be the basis for providing consistency, since this property must be provided in all circumstances, even when the LQP is incorrect.

\subsection{Killijian, Cunningham, Meier, Mazare \& Cahill (2001)}

Killijian et al. (2001) suggest a model for a location-aware group communication system. The key idea is that groups are defined not only by interest but also by proximity. A proximity group therefore consists of members that are within a defined space and that are interested in this group. The space is specified either absolutely or relatively to mobile nodes. For example, an absolute proximity might be defined for a traffic light that is to inform cars about its status. A proximity group around an ambulance, which informs nearby cars about its presence, requires in contrast a relative specification. The group membership management layer is supported by a partition prediction component. This component predicts the probability of a partition and is employed to guarantee consistency of group membership and message delivery. A failure predictor is recommended to predict link failures due to node crashes and battery status. A movement planner predicts mobility related link failures based on the notion of a safe distance (Roman et al. 2001). An environment evaluator is envisioned that infers knowledge from environmental conditions that have an influence on link quality.

While we like the idea of partition prediction, Killijian et al.'s (2001) work provides no details on how such partition prediction can be realized or how such a component integrates into the group membership management layer. Furthermore, only the prediction of link failures is envisioned. However, the prediction of a future network topology also requires a prediction of link recoveries since they also change the network topology. Moreover, Killijian et al. (2001) suggest LQP similar to Roman et al. (2001) for ensuring consistency. As discussed in previously, group communication systems provide consistency guarantees that cannot be realized solely by imperfect LQP.

\section{Handoff algorithms}

Handoff, which is also termed 'handover', is the process of transferring a connection with a mobile phone to a different base station than currently used in a cellular network. This transfer can be realized by switching the frequency band, time slot or codeword used or any combination of these. Handoff algorithms are specific to the structure of the infrastructure networks for which they are designed. For example, an algorithm that is designed to work in a macrocellular environment is unlikely to perform well in an overlay network of macro- 
and microcells. If the infrastructure network controls the handoff process, the handoff is centralized; otherwise it is decentralized. The goal of the handoff process is to maximize communication quality while minimizing the number of handoffs. Handoff, admission control, channel allocation and power control are all resource management tasks that should be integrated to obtain better overall system performance. Most handoff algorithms are based on signal-power and noise-power measurements, which indicate that these criteria are well suited in determining link quality. An excellent survey article for handoff algorithms is given by Tripathi et al. (1998).

The handoff process is related to LQP in MANETs since both topics are concerned with link quality. However, these topics differ greatly in other respects. The handoff process switches between base stations in an infrastructure network. The infrastructure network is static with respect to mobility and therefore has well-defined properties such as certain coverage and fixed positions of the base stations. Furthermore, base stations are elevated, have more powerful transmitters and more sensitive receivers than mobile stations such that up- and down links have different properties. Moreover, base stations are connected by wired links and therefore have global knowledge about all mobile users and other base stations. All these properties are absent in MANETs that consists of mobile nodes, which act as peers only. Furthermore, the aim differs for both topics. The handoff process focuses on selecting the base station with the best communication quality and the accurate prediction of single link failures is of no particular concern. The mobile station can also control the communication quality to a certain extent via the base station due to a feedback loop. The handoff problem is a global optimization problem with global knowledge that aims to simultaneously maximize the communication quality and the capacity of the network. LQP in MANETs, however, aims to predict the probability of link failures without any global knowledge and without the possibility of influencing the communication quality. Furthermore, MANETs require multi-hop communication while cellular networks rely on single-hop wireless communication.

\section{Real-world measurement studies related to link quality prediction}

In recent years an increased number of measurement studies can be found in the literature due to the deployment of wireless networks, especially in universities. However, most of these measurements are concerned with higher-layer metrics in infrastructure networks and indoor studies are predominant. A comprehensive list of measurement studies is given by the 'Community Resource for Archiving Wireless Data At Dartmouth' project that intends to provide a wireless network data resource for the research community (Dartmouth College 2006). In this section we focus on some studies that measure link quality using lowlayer metrics in outdoor 802.11 networks and that are related to our own measurements.

\subsection{Anastasi, Borgia, Conti \& Gregori (2004)}

Anastasi et al. (2004) measured link quality in terms of the higher-level metrics of TCP and UDP throughput for an $802.11 \mathrm{~b}$ network in ad hoc mode. All measurements were done outdoors with static nodes. The authors observed that the maximum transmission range is lower for higher data rates than for lower ones. This result confirms theory since with constant transmission power more energy per symbol is available for lower than for higher 
data rates. The maximum transmission range was estimated to be $30 \mathrm{~m}, 70 \mathrm{~m}, 90-100 \mathrm{~m}$ and 110-130 $\mathrm{m}$ for the data rates of $11 \mathrm{Mbps}, 5.5 \mathrm{Mbps}, 2 \mathrm{Mbps}$ and $1 \mathrm{Mbps}$ respectively. Therefore, Anastasi et al. (2004) conclude (erroneously) that the maximum transmission range of $250 \mathrm{~m}$, which is assumed in most MANET simulations, is 2-3 times higher than in a real-world, obstacle-free environment. Furthermore, it was demonstrated that the throughput is seriously degraded if a node height is below $1.20 \mathrm{~m}$. This effect depends on the First Fresnel Zone that contains most of the radio energy. If nodes are too low, the First Fresnel Zone touches the ground such that a significant amount of radio energy is lost.

Moreover, Anastasi et al. (2004) showed that the UDP and TCP throughput between two nodes was significantly reduced, if other nodes, which were placed further away than the maximum transmission range, transmitted on the same channel. However, the throughput increased in two steps with when the distance between the interfering nodes was increased. Therefore, the authors concluded that the maximum transmission range is smaller than the maximum physical carrier sensing range, which in turn is smaller than the maximum interference range. Consequently, Anastasi et al. (2004) suggest using different values for each of these ranges in simulations that traditionally use only one value for all ranges.

\subsection{Aguayo, Bicket, Biswas, Judd \& Morris (2004)}

Aguayo et al. (2004) measured packet loss for a static mesh network of 38 nodes that were distributed over roofs in Cambridge, Massachusetts. Each node consisted of a PC with an 802.11b card with Intersil Prism 2.5 chipset in ad hoc mode and an omni-directional antenna. All measurements of signal power, noise power and packet loss were made with 1500-byte long packets at different data rates. The accuracy of the signal power and noise power measurements was shown to be within $4 \mathrm{~dB}$ for the type of 802.11 card used.

The 'neighbour' abstraction is commonly used in the design of MANET protocols and it expresses the idea that nodes can be grouped into partitions in which nodes can either communicate with $100 \%$ or $0 \%$ probability. However, Aguayo et al. (2004) demonstrated that a high number of nodes had packet loss ratios within the range of $10 \%$ to $90 \%$. Therefore, the classical neighbour abstraction did not hold for this wireless test bed. Furthermore, it was shown that the observed packet loss was only partly depending on the signal-to-noise ratio (SNR) or distance. In an experiment with a hardware channel emulator it was determined that a delay spread higher than a few hundred nanoseconds affected the packet loss negatively. This observation agrees with the 802.11 cards' specification which states that the RAKE receiver, which is the main receiver structure in an 802.11 card, and the channel equalizer only support delay spreads up to $250 \mathrm{~ns}$. While this delay spread is appropriate for indoor environments, urban microcells exhibit delay spread often exceeding $1 \mathrm{~ms}$ (Aguayo et al. 2004). Moreover, it was shown that the packet loss typically varies by only a few per cent within a time frame of one second.

While Aguayo et al. (2004) present interesting results, it is not clear how general these are. All their measurements have been made with only one card type. The propagation environment is over urban roofs in which diffraction is especially high. If the same observations would be made on the ground for moving nodes is open to question. Additionally, all measurements were made with very long packets of only one size and therefore it is open if the same results could be observed with different packet lengths. 


\subsection{Sridhara, Shin \& Bohacek (2006)}

Sridhara et al. (2006) examined signal-power variations due to pedestrians for an 802.11 network in infrastructure mode. Two nodes were placed at heights of $1.2 \mathrm{~m}$ and $4.3 \mathrm{~m}$ respectively and the signal power was simultaneously recorded for both transmitting nodes. It was observed that the signal power varied due to moving pedestrians albeit all nodes were static. Sridhara et al. (2006) noticed further that with increasing density of pedestrians the variance of the signal power increased. Different node heights, however, only slightly affected the variance of the signal power. Moreover, it was demonstrated that the variations can be characterized well in the time domain by a one-dimensional diffusion process with four parameters. This statistical description allows a computationally inexpensive simulation of the observed variations.

The proposed statistical description of signal-power variations, which are caused by pedestrians, is valid for stationary nodes only. Whether these results extend to mobile scenarios has not been explored yet. Furthermore, the authors do not investigate to what degree these signal-power fluctuations are caused by small-scale fading. Small-scale fading can also be experienced by static nodes if other objects move in the radio channel (Rappaport 2002, p. 178).

\section{Small-scale wideband signal envelope fading}

In general the wideband signal envelope shows less severe small-scale fading than the narrowband signal envelope (Lee 1991). The reason for this is that wideband signals inherently exploit frequency diversity (Lee 1991, Kozono 1994, Yamaguchi et al. 1995). While the probability distributions of small-scale narrowband signal envelope fading, for which closed-form expressions exist, have been extensively studied (see, for example, Young 1952, Nakagami 1960, Jakes \& Reudink 1967, Clarke 1968, Suzuki 1977), comparatively few such studies are reported for wideband systems (Kozono 1994, Yamaguchi et al. 1995, Yan \& Kozono 1999, Oh et al. 2001) and closed-form expressions for the CDF and PDF of the small-scale fading envelope are unavailable.

\subsection{Kozono (1994) and Yan \& Kozono (1999)}

Kozono (1994) and Yan \& Kozono (1999) have proposed a wideband signal propagation model with which they investigated the small-scale fading of the received signal envelope of a mobile receiver. They verified their model with extensive measurements performed in the Tokyo region (Kozono 1994, Nakabayashi \& Kozono 1998, Yan \& Kozono 1999, Nakabayashi et al. 2001) and observed that the fading depth is strongly dependent not only on the ratio of the direct to indirect power of the wideband signal but also on what they term the 'Equivalent Received Bandwidth'. They also observed that the distribution of the signal envelope is almost independent of the carrier frequency. Their results also demonstrate that wideband signals in general exhibit shallower fades than narrowband signals and that small-scale wideband signal envelope fading in non-line-of sight (NLOS) conditions cannot in general be characterized using the Rayleigh distribution. They did not investigate however whether other known distributions can do this. Similar results to those given above were reported by Yamaguchi et al. (1995). 
Yan \& Kozono's (1999) model assumes that $\mathrm{N}_{\mathrm{wv}}$ multipath waves arrive at the receiver under the following conditions: each wave has an amplitude $A_{i}$, a path length $L_{i}$ and an angle of arrival $\vartheta_{\mathrm{i}} . \mathrm{A}_{\mathrm{i}}$ and $\mathrm{L}_{\mathrm{i}}$ are independent of each other and are distributed uniformly over a given range. $\vartheta_{\mathrm{i}}$ is distributed uniformly over $2 \pi$ in the horizontal plane. $\mathrm{A}_{0}$ and $\mathrm{L}_{0}$ denote the amplitude and path length of the LOS wave. The ratio of the direct to indirect power is defined as:

$$
\mathrm{a}=\mathrm{A}_{0}^{2} / \sum_{\mathrm{i}=1}^{\mathrm{N}_{W_{\mathrm{v}}}-1} \mathrm{~A}_{\mathrm{i}}^{2}
$$

The bandwidth of each arriving wave (assumed to have a flat power spectral density) is taken to be greater than the receiver bandwidth $2 \Delta \mathrm{f}$. The received signal power $\mathrm{R}$ in watts is then expressed by (Yan \& Kozono 1999, (2)):

$$
\mathrm{R}(2 \Delta \mathrm{f})=2 \Delta \mathrm{f}(\sum_{\mathrm{i}=0}^{N_{W_{v}}-1} \mathrm{~A}_{\mathrm{i}}^{2}+\underbrace{\sum_{\mathrm{i}=0}^{N_{W_{v}}}-1 \sum_{\mathrm{j}=0}^{N_{W_{v}}-1} \frac{\mathrm{c}}{2 \pi} \frac{\mathrm{A}_{\mathrm{i}} \mathrm{A}_{\mathrm{j}}}{\Delta \mathrm{f} \Delta \mathrm{L}_{\mathrm{ij}}} \cos \left(\frac{2 \pi \mathrm{f}_{\mathrm{c}} \Delta \mathrm{L}_{\mathrm{ij}}}{\mathrm{c}}\right) \sin \left(\frac{2 \pi \mathrm{f}_{\mathrm{c}} \Delta \mathrm{L}_{\mathrm{ij}}}{\mathrm{c}}\right)}_{\mathrm{i} \neq \mathrm{j}})
$$

where $\Delta \mathrm{L}_{\mathrm{ij}}$ is the difference in the path lengths of the ith and $\mathrm{jth}$ arriving waves. As mentioned earlier Yan \& Kozono (1999) also propose a propagation parameter termed the 'equivalent received bandwidth' which can be used to estimate the fading depth (Cardoso \& Correia 2003). The equivalent received bandwidth is the product of the receiver bandwidth $2 \Delta \mathrm{f}$ and the maximum difference in path lengths $\Delta \mathrm{L}_{\max }$ of the arriving waves (i.e. $\Delta \mathrm{L}_{\max }=\max \left|\mathrm{L}_{\mathrm{i}}-\mathrm{L}_{\mathrm{j}}\right|$ ). In summary, the equivalent bandwidth and hence observed fading depth are dependent on the receiver bandwidth and the propagation environment.

\subsection{Nakabayashi \& Kozono (1998)}

Two properties of the stochastic process of small-scale wideband signal envelope fading are important: the probability distribution of the process and its autocorrelation. The probability distribution is the summary of the amplitude structure of the process; the autocorrelation is the summary of the time structure (Yates \& Goodman 2005, p. 353). Nakabayashi \& Kozono (1998) investigated the autocorrelation properties of small-scale wideband signal envelope fading based on Kozono's (1994) wideband signal propagation model. It was shown that the normalized autocorrelation of the small-scale fading signal envelope $r$ is independent of the receiver bandwidth and is described for both, narrowband and wideband signals, by (Nakabayashi \& Kozono 1998, (2)), (Goldsmith 2005, pp. 73,74):

$$
\rho_{\mathrm{rr}}\left(\Delta_{\mathrm{t}}\right)=\mathrm{J}_{0}\left(2 \pi \mathrm{f}_{\mathrm{D}} \Delta_{\mathrm{t}}\right)
$$

where $f_{D}$ is the Doppler frequency, $\Delta_{t}$ is the time lag of the normalized autocorrelation, and $\mathrm{J}_{0}$ is the zeroth-order Bessel function of the first kind. This result was derived analytically and also verified by measurements (Nakabayashi \& Kozono 1998). 


\subsection{Oh, Lee, Choi \& Kim (2001)}

In their work on a statistical model of a W-CDMA receiver, Oh et al. (2001) showed that the distribution of the small-scale fading signal envelope can be described using the Nakagami distribution for a CDMA system. Though this simulation study was limited to only two receiver bandwidths deployed in two types of urban environment, namely, urban high-rise and urban residential, it suggested to us the possibility that the Nakagami and the closely related Rice distribution could be used as a general means with which to describe the distribution of the small-scale fading wideband signal envelope, albeit to a certain degree of approximation.

\section{Accuracy and methodology of MANET simulations}

Simulation is still the most popular means of evaluation in the MANET community (Kurkowski et al. 2005) since real-world measurements are expensive in terms of labour and equipment. Simulations also allow the influence of parameters on evaluation metrics to be isolated and simulation experiments are easy to repeat. Nevertheless, the accuracy of current MANET simulators is debatable as some studies suggest. Furthermore, the methodology of MANET studies is often questionable and seems to be poorer than in more established fields (Kurkowski et al. 2005). Therefore, we discuss here some key studies that enabled credible evaluations in this work.

\subsection{Cavin, Sasson \& Schiper (2002)}

Cavin et al. (2002) implemented a simple broadcast algorithm to compare the basic metrics of this protocol in three widely used MANET simulators, namely GloMoSim, NS-2 and OPNET without further assessment of these results in real-world experiments. The used broadcast algorithm floods the network by forwarding every message received for the first time to neighbouring nodes. This algorithm is simple enough to be implemented equally with the same parameters in all three simulators while also being relevant since broadcasting is an important building block in MANET protocols. Cavin et al. (2002) demonstrated that the evaluation results were quantitatively and qualitatively divergent. Not only did the absolute values of the evaluation metrics differ but also in some cases the general behavior varied. They explained these observations by the different choice of models and different level of detail in the simulators used, especially in the physical layer. Similar results were obtained by Takai et al. (2001) who showed that the AODV and DSR routing protocols lead to different evaluation results in NS-2 than in GloMoSim, mainly due to differences in the physical layer. Both studies, as well as our own real-world measurements, lead us to the conclusion that the standard NS-2 simulator is unsuitable for credible evaluations.

\subsection{Gray, Kotz, Newport, Dubrovsky, Fiske, Liu, Masone, McGrath \& Yuan (2004)}

Gray et al. (2004) conducted a study in which they compared the performance of four ad hoc routing protocols in an outdoor trial, an indoor trial and simulations. The network consisted of 33 laptops that were equipped with an 802.11 card and a GPS receiver each. In the outdoor trial, people moved randomly, similar to the Random Waypoint Mobility Model with no pauses, and the nodes would broadcast their GPS positions every three 
seconds. The locations were saved in files and a connectivity trace was derived that represents the network topology. Two nodes were modeled to be connected within the beacon interval, if they received a beacon; otherwise they were modeled to be disconnected. The experiment occurred on an athletic field that can roughly be divided into four flat, equally sized sections of which one was approximately $4 \mathrm{~m}$ to $6 \mathrm{~m}$ lower. This difference in height caused blockage in LOS paths. In the indoor trials, all laptops were located on the same tabletop and the GPS and connectivity traces were used to emulate mobility and connectivity. In the simulation experiment, six different combinations of radio propagation models and the connectivity trace were compared. The Free Space Propagation Model, the Two-Ray Ground Propagation Model and a singleslope path-loss model with an empirically derived path-loss exponent and large-scale fading were used either with or without the connectivity trace. If the connectivity trace was enabled, the simulator would first check that nodes were in range according to the trace file before the radio propagation model calculated the received power. If the power was higher than a threshold, a packet is received; otherwise it is lost. SWAN (Perrone \& Nicol 2002) was the simulator of choice and the mobility was given by the GPS trace file.

Gray et al. (2004) demonstrated that the indoor experiments did not represent the outdoor behavior despite relying on the outdoor connectivity trace. The performance metrics differed in values and even the relative ranking between the routing protocols varied. This observation was attributed mainly to interference that was caused by the 33 nodes all being located on the same tabletop. The comparison of simulations with outdoor measurements showed that the single-slope path-loss model with large-scale fading was fairly accurately predicting the outdoor performance while the other model combinations led to poorer representations of the real-world environment. This result indicated to us that stochastic radio propagation models with empirical constants for path loss and large-scale fading can lead to accurate outdoor simulations of MANETs, especially if these models are extended with simulation of wideband small-scale fading.

\subsection{Stepanov, Herrscher \& Rothermel (2005)}

Stepanov et al. (2005) compared the NS-2 simulator with a single-slope path-loss model (without any fading simulation) to an augmented NS-2 version that calculates the received power at nodes via a ray tracing based radio propagation model. The latter type of radio propagation model is known to describe the radio channel accurately when detailed geographical maps with building materials are used (Neskovic et al. 2000). Stepanov et al. (2005) demonstrated that the network topology differed significantly between simulations with the single-slope path-loss model with different constants and the ray tracing model. Additionally, it was shown that the packet delivery ratio, the routing packet overhead and the packet latency differed for simulations of the AODV protocol. Unfortunately, no comparison was made between the ray tracing model and a single-slope path-loss model with additional simulation of large-scale and small-scale fading.

For their study, Stepanov et al. (2005) precomputed the signal power for all possible transmitter-receiver positions in the city centre of Stuttgart with a commercial ray tracing based radio propagation simulator. This precomputed data was then fetched during 
simulations in NS-2. The signal power was precomputed for a $5 \mathrm{~m}$ by $5 \mathrm{~m}$ grid that leads to 32 billion position pairs for the city centre of Stuttgart. A finer sampling would have lead to significantly more position pairs. The precalculation step produced 120 GB of data and required the computing power of three days of a PC cluster of 50 machines. These required resources indicated clearly to us that ray traced based methods do not scale to ad hoc networks. While in infrastructure networks the position of base stations is fixed, every node in ad hoc networks is potentially mobile such that the problem of precomputing signal-power values increases by a whole dimension. Consequently, an improved stochastic simulation of the radio channel is better suited to the simulation of MANETs due to significantly lower resource requirements than using ray tracing based methods.

\subsection{Kurkowski, Camp \& Colagrosso (2005)}

Kurkowski et al. (2005) investigated the methodology on which the MANET community performs evaluations. Their findings are derived by surveying all papers that have been published in the premier conference MobiHoc from 2000 to 2005. Four different criteria were identified for credible research. First, results should be repeatable. Secondly, results should be unbiased and not specific to an unrepresentative scenario. Thirdly, results should be rigorous and therefore scenarios and conditions of simulations should truly represent the aspects of the MANET protocol being studied. Fourthly, the results must be statistically sound. Based on these criteria, Kurkowski et al. (2005) concluded that the state of art in MANET simulations is poor and that most studies lack believability.

$75.5 \%$ of all surveyed studies were based on simulations. Nevertheless, in $29.8 \%$ of all studies the simulator was not identified. NS-2, followed by GloMoSim, was by far the most popular MANET simulator and NS-2/GloMoSim were used in $43.8 \% / 10.0 \%$ of all studies in which the simulator was identified. $57.9 \%$ of all studies did not state the type of simulation (e.g. termination or steady-state). Steady-state simulations represent the long-term behavior of MANET protocols after the initialization phase and they are the most common type of simulations since researchers are normally interested in the long-term behavior of protocols. However, only $7.0 \%$ of all studies addressed the initialization bias albeit using the unreliable method of arbitrarily discarding data from the beginning of the simulation. The number of simulation iterations was only published in $35.8 \%$ of all surveyed studies and confidence intervals were provided only in $12.5 \%$. However, it is unclear how well results represent the population under study when the number of simulation iterations and confidence intervals are unknown.

\subsection{PalChaudhuri, Boudec \& Vojnovic (2005)}

Steady-state simulations of MANETs require that the mobility distribution is in steady state within the whole simulation run since protocol performance varies significantly between the transient phase and the steady state as, for example, PalChaudhuri et al. (2005) showed for the packet reception ratio of the DSR protocol. Steady-state simulation of mobility, which is also called perfect simulation, is provided for the random trip model by a tool that was developed by PalChaudhuri et al. (2005). The random trip model is a generic model that includes the widespread Random Waypoint Mobility Model (Johnson \& Maltz 1996) and also the Random 
Waypoint Mobility Model City Section (Saha \& Johnson 2004). In the latter model the nodes move according to a geographical map that is often derived from the real world such that this mobility model is often considered to be realistic (Saha \& Johnson 2004).

\section{Summary}

All the surveyed link quality prediction (LQP) algorithms are based on radio propagation models that only characterize path loss to various degrees of accuracy but fail to also consider large-scale and wideband small-scale fading effects. Consequently, neither these LQP algorithms themselves nor their performance evaluations are applicable to real-world environments in which these two propagation effects are pronounced (Neskovic et al. 2000, Kotz et al. 2004). Hence, novel LQP algorithms are required that incorporate these propagation effects and make the promise of accurate LQP in real-world urban environments a reality. Furthermore, the need for credible evaluations by real-world studies or advanced simulations, which account for the propagation effects of path loss, large-scale and small-scale fading, is evident.

A number of LQP algorithms rely on location measurements for their prediction. However, typical GPS measurements have a user-equivalent range error of $19.2 \mathrm{~m}$ and location information is often unobtainable in dense urban environments where a connection with at least three satellites cannot be continuously maintained. Therefore, location measurements are currently impractical for these environments.

In the literature indirect evaluations of LQP algorithms prevail over direct ones. In these indirect evaluations the prediction accuracy of LQP algorithms is assessed by comparing the performance of communication protocols with and without LQP, typically by comparing metrics such as packet latency and data throughput. Since various studies use different communication protocols to evaluate their LQP algorithms, a direct comparison of LQP accuracy for different studies is difficult.

Link quality prediction is currently in its nascent stage. It is fundamentally a propagation problem which applies not just to ad-hoc networks per se but to cognitive radio in general where it is necessary to be able to calculate the electromagnetic footprint of a cognitive radio in order to guarantee non-interference. In conclusion an effort, similar to that seen forty years ago with the dawn of cellular radio in propagation modeling for network planning, must now take place in real-time radio propagation prediction.

\section{References}

Agarwal, S., Ahuja, A., Singh, J. P. \& Shorey, R. (2000), Route-Lifetime Assessment Based Routing (RABR) Protocol for Mobile Ad-Hoc Networks, in 'IEEE International Conference on Communications', Vol. 3, New Orleans, LA, USA, pp. 1697-1701.

Aguayo, D., Bicket, J., Biswas, S., Judd, G. \& Morris, R. (2004), Link-Level Measurements from an 802.11b Mesh Network, in 'ACM SIGCOMM Conference on 
Communications Architectures, Protocols and Applications', Vol. 4, Portland, OR, USA.

Anastasi, G., Borgia, E., Conti, M. \& Gregori, E. (2003), IEEE 802.11 Ad Hoc Networks: Performance Measurements, in 'Workshop on Mobile and Wireless Networks', Providence, RI, USA.

Bajaj, L., Takai, M., Ahuja, R., Tang, K., Bagrodia, R. \& Gerla., M. (1999), Glomosim: A Scalable Network Simulation Environment., Technical report, UCLA Computer Science Department.

Cavin, D., Sasson, Y. \& Schiper, A. (2002), On the Accuracy of MANET Simulators, in 'Workshop on Principles of Mobile Computing', Toulouse, France, pp. 38-43.

Clarke, R. H. (1968), 'Statistical Theory of Mobile-Radio Reception', Bell System Technical Journal 47(6), 957-1000.

Cardoso, F. D. \& Correia, L. M. (2003), 'Fading Depth Dependence on System Bandwidth in Mobile Communications - an Analytical Approximation', IEEE Transactions on Vehicular Technology 52(3).

Dartmouth College (2006), 'Community Resource for Archiving Wireless Data at Dartmouth'. http://crawdad.cs.dartmouth.edu/

De Couto, D. S. J., Aguayo, D., Bicket, J. \& Morris, R. (2003), A High-Throughput Path Metric for Multi-Hop Wireless Routing, in 'International Conference on Mobile Computing and Networking', San Diego, CA, USA, pp. 134-146.

Dowling, J., Curran, E., Cunningham, R. \& Cahill, V. (2005), 'Using Feedback in Collaborative Reinforcement Learning to Adaptively Optimize MANET Routing', IEEE Transactions on Systems, Man and Cybernetics 35(3), 360-372.

Dube, R., Rais, C. D., Wang, K.-Y. \& Tripathi, S. K. (1997), 'Signal Stability-Based Adaptive Routing (SSA) for Ad Hoc Mobile Networks', IEEE Personal Communications 4(1), 36-45.

Fischer, M. J., Lynch, N. A. \& Paterson, M. S. (1985), 'Impossibility of Distributed Consensus with One Faulty Process', Journal of the ACM 32(2), 374-382.

Gerharz, M., Waal, C. d., Frank, M. \& Martini, P. (2002), Link Stability in Mobile Wireless Ad Hoc Networks, in 'IEEE Conference on Local Computer Networks', Tampa, FL, USA, pp. 30-42.

Gerla, M., Pei, G., Lee, S.-J. \& Chiang, C.-C. (1998), ‘On-Demand Multicast Routing Protocol (ODMRP) for Ad Hoc Networks'. Internet Draft, draft-ietf-manet-odmrp-00.txt, Nov. 1998, work in progress.

Gerla, M., Tang, K. \& Bagrodia, R. (1999), TCP Performance in Wireless Multi-Hop Networks, in 'Workshop on Mobile Computer Systems and Applications', New Orleans, LA, USA, pp. 41-50.

Goff, T., Abu-Ghazaleh, N. B., Phatak, D. S. \& Kahvecioglu, R. (2001), Preemptive Routing in Ad Hoc Networks, in 'International Conference on Mobile Computing and Networking', Rome, Italy, pp. 43-52.

Goldsmith, A. (2005), Wireless Communications, 1st edn, Cambridge University Press.

Gray, R. S., Kotz, D., Newport, C., Dubrovsky, N., Fiske, A., Liu, J., Masone, C., McGrath, S. \& Yuan, Y. (2004), Outdoor Experimental Comparison of Four Ad Hoc Routing 
Algorithms, in 'International Workshop on Modeling Analysis and Simulation of Wireless and Mobile Systems', Venice, Italy, pp. 220-229.

Grewal, M. S., Weill, L. R. \& Andrews, A. P. (2001), Global Positioning Systems, Inertial Navigation and Integration, John Wiley and Sons.

Harvey, A. C. (1993), Time Series Models, 2nd edn, The MIT Press.

Hines, W. W., Montgomery, D. C., Goldsman, D. M. \& Borror, C. M. (2002), Probablity and Statistics in Engineering, 4th edn, John Wiley and Sons.

Holland, G. \& Vaidya, N. (2002), 'Analysis of TCP Performance over Mobile Ad Hoc Networks', Wireless Networks 8(2/3), 275-288.

Jakes, W.C., J. \& Reudink, D. (1967), 'Comparison of Mobile Radio Transmission at UHF and X-Band', IEEE Transactions on Vehicular Technology 16, 10-13.

Jiang, S., He, D. \& Rao, J. (2005), 'A Prediction-Based Link Availability Estimation for Routing Metrics in MANETs', IEEE/ACM Transactions on Networking 13(6), 1302 1312.

Johnson, D. \& Maltz, D. (1996), Dynamic Source Routing in Ad Hoc Wireless Networks, in Imelinsky \& H. Korth, eds, 'Mobile Computing', Kluwer Academic Publishers, pp. 153-181.

Kay, D. A. (2001), Trigonometry, 1st edn, Cliffs Notes.

Killijian, M.-O., Cunningham, R., Meier, R., Mazare, L. \& Cahill, V. (2001), Towards Group Communications for Mobile Participants, in 'ACM Workshop on Principles of Mobile Computing', Newport, RI, USA.

Kotz, D., Newport, C., Gray, R. S., Liu, J., Yuan, Y. \& Elliott, C. (2004), Experimental Evaluation of Wireless Simulation Assumptions, in 'International Workshop on Modeling Analysis and Simulation of Wireless and Mobile Systems', Venice, Italy, pp. 78-82.

Kozono, S. (1994), 'Received Signal-Level Characteristics in a Wide-Band Mobile Radio Channel', IEEE Transactions on Vehicular Technology 43(3).

Kurkowski, S., Camp, T. \& Colagrosso, M. (2005), 'MANET Simulation Studies: The Incredibles', ACM SIGMOBILE Mobile Computing and Communications Review 9(4), 50-61.

Lee, W. C. Y. (1991), Theory of Wideband Radio Propagation, in 'IEEE Vehicular Technology Conference', St. Louis, MO, USA.

Lee, S.-J., Su, W. \& Gerla, M. (1999), Ad Hoc Wireless Multicast with Mobility Prediction, in 'IEEE International Conference on Computer Communications and Networks', Boston, MA, USA, pp. 4-9.

McDonald, A. B. \& Znati, T. (1999a), 'A Mobility-Based Framework for Adaptive Clustering in Wireless Ad-Hoc Networks', IEEE Journal on Selected Areas in Communication 17(8).

McDonald, A. B. \& Znati, T. (1999b), A Path Availability Model for Wireless Ad-Hoc Networks, in 'IEEE Wireless Communications and Networking Conference', New Orleans, LA, USA.

MIL 3 Inc. (2006), ‘OPNET’. http:/ / www.opnet.com. 
Nakabayashi, H. \& Kozono, S. (1998), 'Autocorrelation Characteristics for Received SignalLevel in a Wide-Band in a Wide-Band Mobile Radio Channel', Transactions IEICE J81-B-II(2).

Nakabayashi, H., Yan, J., Masui, H., Ishii, M., Sakawa, K., Shimizu, H., Kobayashi, T. \& Kozono, S. (2001), 'Validation of Equivalent Received Bandwidth to Characterize Received Signal Level Distribution through Experiment and Simulation', IEICE Transactions on Communications E84-B(9), 2550-2559.

Nakagami, M. (1960), The M-Distribution; a General Formula of Intensity of Rapid Fading, in W. G. Hoffman, ed., 'Statistical Methods in Radio Wave Propagation: Proceedings of a Symposium held at the University of California', Permagon Press, pp. 3-36.

Neskovic, A., Neskovic, N. \& Paunovic, G. (2000), 'Modern Approaches in Modeling of Mobile Radio Systems Propagation Environment', IEEE Communications Surveys 3(3), 2-12.

Oh, D., Lee, G., Choi, D. \& Kim, C. (2001), Statistical Model of W-CDMA Signals over Realistic Multipath Channel, in 'Vehicular Technology Conference', Vol. 1, Rodes, Greece, pp. 347-351.

PalChaudhuri, S., Boudec, J.-Y. L. \& Vojnovic, M. (2005), Perfect Simulations for Random Trip Mobility Models, in 'Annual Simulation Symposium', San Diego, CA, USA, pp. 72-79.

Paul, K., Bandyopadhyay, S., Mukherjee, A. \& Saha, D. (1999), Communication-Aware Mobile Hosts in Ad-Hoc Wireless Network, in 'International Conference on Personal Wireless Communications', Jaipur, India, pp. 83-87.

Perkins, C. E. \& Bhagwat, P. (1994), Highly Dynamic Destination-Sequenced Distance-Vector Routing (DSDV) for Mobile Computers, in 'ACM SIGCOMM Conference on Communications Architectures, Protocols and Applications', London, UK, pp. 234244.

Perkins, C., Royer, E. \& Das, S. (2001), 'Ad Hoc on-Demand Distance Vector (AODV) Routing'. Internet Draft, Internet Engineering Task Force, Mar. 2001.

http://www.ietf.org/internet-drafts/draft-ietf-manet-aodv-08.txt

Perrone, L. F. \& Nicol, D. M. (2002), A Scalable Simulator for TinyOS Applications, in 'Winter Simulation Conference', San Diego, CA, USA.

Polya, G. (1921), 'Ueber eine Aufgabe der Wahrscheinlichkeitstheorie betreffend die Irrfahrt im Strassennetz', Mathematische Annalen 84(1-2), 149-160.

Punnoose, R. J., Nikitin, P. V., Broch, J. \& Stancil, D. D. (1999), Optimizing Wireless Network Protocols Using Real-Time Predictive Propagation Modeling, in 'IEEE Radio and Wireless Conference', Denver, CO, USA.

Rappaport, T.S. (2002), Wireless Communications - Principles and Practice, Prentice Hall Communications Engineering and Emerging Technologies Series, 2nd edn, Prentice Hall.

Qin, L. \& Kunz, T. (2002), 'Pro-Active Route Maintenance in DSR', ACM SIGMOBILE Mobile Computing and Communications Review 6(3), 79-89. 
Qin, L. \& Kunz, T. (2003), Increasing Packet Delivery Ratio in DSR by Link Prediction, in '36th Annual Hawaii International Conference on System Sciences', Vol. 9, Hawaii, USA.

Roman, G.-C., Huang, Q. \& Hazemi, A. (2001), Consistent Group Membership in Ad Hoc Networks, in 'IEEE International Conference in Software Engineering', Toronto, Canada, pp. 381-388.

Saha, A. K. \& Johnson, D. B. (2004), Modeling Mobility for Vehicular Ad Hoc Networks, in 'Workshop on Vehicular Ad Hoc Networks (VANET)', Philadelphia, PA, USA.

Schiller, J. (2000), Mobile Communications, 1st edn, Addison-Wesley Professional

Sridhara, V., Shin, H.-C. \& Bohacek, S. (2006), Observations and Models of Time-Varying Channel Gain in Crowded Areas, in 'Workshop on Wireless Network Measurements', Boston, MA, USA.

Stepanov, I., Herrscher, D. \& Rothermel, K. (2005), On the Impact of Radio Propagation Models on MANET Simulation Results, in 'IFIP International Conference on Mobile and Wireless Communication Networks', Marrakech, Morocco.

Suzuki, H. (1977), 'A Statistical Model for Urban Radio Propagation', IEEE Transactions on Communications 25(7), 673-680.

Takai, M., Martin, J. \& Bagrodia, R. (2001), Effects of Wireless Physical Layer Modeling in Mobile Ad Hoc Networks, in 'International Conference on Mobile Computing and Networking', Long Beach, CA, USA, pp. 87-94.

Tobagi, F. \& Kleinrock, L. (1975), 'Packet Switching in Radio Channels: Part II - the Hidden Terminal Problem in Carrier Sense Multiple-Access and the Busy-Tone Solution', IEEE Transactions on Communications 23(12).

Tripathi, N. D., Reed, J. H. \& Van Landingham, H. F. (1998), 'Handoff in Cellular Systems', IEEE Personal Communications 5(6), 26-37.

Wang, Y., Martonosi, M. \& Peh, L.-S. (2006), A Supervised Learning Approach for Routing Optimizations in Wireless Sensor Networks, in 'REALMAN', Florence, Italy, pp. 79-86.

Woo, A., Tong, T. \& Culler, D. (2003), Taming the Underlying Challenges of Reliable Multihop Routing in Sensor Networks, in 'International Conference on Embedded Networked Sensor Systems', Los Angeles, CA, USA, pp. 14-27.

$\mathrm{Xu}$, S. \& Saadawi, T. (2001), 'Does the IEEE 802.11 MAC Protocol Work Well in Multihop Wireless Ad Hoc Networks?', IEEE Communications Magazine 39(6), 130-137.

Yamaguchi, A., Suwa, K. \& Kawasaki, R. (1995), Received Signal Level Characteristics for Wideband Radio Channel in Microcells, in 'International Symposium on Personal, Indoor and Mobile Radio Communications', Toronto, Canada.

Yan, J. \& Kozono, S. (1999), 'A Study of Received Signal-Level Distribution in Wideband Transmissions in Mobile Communications', IEEE Transactions on Vehicular Technology 48(5).

Yates, R. D. \& Goodman, D. J. (2005), Probability and Stochastic Processes, 2nd edn, John Wiles and Sons. 
Young, W.R., J. (1952), 'Comparison of Mobile Radio Transmission at 150, 450, 900, and 3700 MC', Bell System Technical Journal 31(6), 1068-1085. 


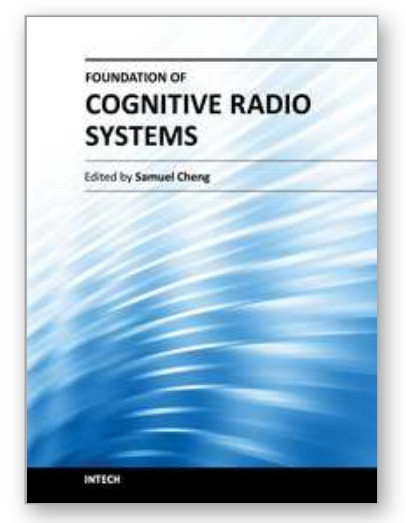

\author{
Foundation of Cognitive Radio Systems \\ Edited by Prof. Samuel Cheng
}

ISBN 978-953-51-0268-7

Hard cover, 298 pages

Publisher InTech

Published online 16, March, 2012

Published in print edition March, 2012

The fast user growth in wireless communications has created significant demands for new wireless services in both the licensed and unlicensed frequency spectra. Since many spectra are not fully utilized most of the time, cognitive radio, as a form of spectrum reuse, can be an effective means to significantly boost communications resources. Since its introduction in late last century, cognitive radio has attracted wide attention from academics to industry. Despite the efforts from the research community, there are still many issues of applying it in practice. This books is an attempt to cover some of the open issues across the area and introduce some insight to many of the problems. It contains thirteen chapters written by experts across the globe covering topics including spectrum sensing fundamental, cooperative sensing, spectrum management, and interaction among users.

\title{
How to reference
}

In order to correctly reference this scholarly work, feel free to copy and paste the following:

Gregor Gaertner and Eamnn O'Nuallain (2012). Link Quality Prediction in Mobile Ad-Hoc Networks, Foundation of Cognitive Radio Systems, Prof. Samuel Cheng (Ed.), ISBN: 978-953-51-0268-7, InTech, Available from: http://www.intechopen.com/books/foundation-of-cognitive-radio-systems/link-quality-predictionfor-mobile-ad-hoc-networks

\section{INTECH}

open science | open minds

\author{
InTech Europe \\ University Campus STeP Ri \\ Slavka Krautzeka 83/A \\ 51000 Rijeka, Croatia \\ Phone: +385 (51) 770447 \\ Fax: +385 (51) 686166 \\ www.intechopen.com
}

\author{
InTech China \\ Unit 405, Office Block, Hotel Equatorial Shanghai \\ No.65, Yan An Road (West), Shanghai, 200040, China \\ 中国上海市延安西路 65 号上海国际贵都大饭店办公楼 405 单元 \\ Phone: +86-21-62489820 \\ Fax: +86-21-62489821
}


(C) 2012 The Author(s). Licensee IntechOpen. This is an open access article distributed under the terms of the Creative Commons Attribution 3.0 License, which permits unrestricted use, distribution, and reproduction in any medium, provided the original work is properly cited. 